This is the authors' personal post-print.

Published in Engineering Structures 101 (2015) 412-426

For the publisher's official offprint please visit:

http://www.sciencedirect.com/science/article/pii/S0141029615004654 or use the DOI identifier:

10.1016/j.engstruct.2015.07.022 


\title{
Dynamic analysis of a beam on block-and-tackle suspension system: A continuum approach
}

\author{
Attila Kocsis, Róbert K. Németh, Baigalimaa Turmunkh \\ Department of Structural Mechanics, Budapest University of Technology and Economics
}

\begin{abstract}
In this paper the dynamic analysis of a beam on a block-and-tackle suspension system is accomplished using a continuum approach. The modal shape functions and the natural frequencies of the structure are derived in a dimensionless form for both slacked and stressed cables. A procedure is developed to handle the nonlinearity originated from the consecutive slacking and stressing of the suspension cable. Vibration analysis of the bilinear, multi-degree-of-freedom structure is accomplished for a vortex-shedding generated lift force and for a continuous pedestrian flow.
\end{abstract}

Keywords: block-and-tackle suspension system, cable slacking, modal shape functions, non-linear dynamics, vortex-shedding, pedestrian load

\section{Nomenclature}

$u(\xi, \tau)$ dimensionless beam deflection

$\xi$ dimensionless coordinate

$\tau$ dimensionless time

$\mu$ beam mass per unit length

EI bending stiffness

$L$ total length of the beam

$r$ frequency parameter

$q(\xi, \tau)$ distributed load

$q_{\mathrm{s}}$ static distributed load intensity

$q_{0}$ dynamic distributed load amplitude

$f$ forcing frequency
$\mathbf{T}$ transformation from active to passive modes

$T_{f}$ period of vibration of the forced structure

$T$ reference time period $(1 / f)$

$T_{\mathrm{n}, i}$ dimensionless natural period*

$f_{i}$ natural frequency*

$\lambda_{i}$ eigenparameter*

$u_{i}(\xi)$ normalized modal shape function*

$F_{i}$ area of the $i$ th normalized shape function*

$R_{\mathrm{d}, i}$ deformation response factor of mode $i^{*}$

$\eta_{i}$ modal displacement*

* Superscript "a" or "p" would correspond to active or passive suspension system, respectively.

Email address: kocsis@ep-mech.me.bme.hu (Attila Kocsis) 


\section{Introduction}

Simple suspension bridges were already used more than 1000 years ago. The oldest known structure is from the 7th century, constructed by the Maya civilization at Yaxchilan [1]. Sketches of the first suspension bridge that resembles modern suspension and cable-stayed bridges appeared in Fausto Veranzio's masterwork [2] in the late 15th century. These type of structures are composed of compressed pillars, a bridge deck, and cables. The main idea behind a suspension bridge is that there are (usually) two main cables that hang between the pillars and are anchored to the ground at both ends, while (vertical or inclined) suspenders connect the deck to the main cables. The cablestayed bridges, on the other hand, have one or more pillars that are the main load bearing structures and inclined suspension cables transmit forces from the deck to the pillars. There are numerous variations of these kind of structures, see for example the comprehensive work of Kawada [3]. The length of suspension bridges varies from small footbridges, like the Boston Public Garden Footbridge, to the Akashi Kaikyo Bridge, whose central span is almost $2000 \mathrm{~m}$ long.

Longer and more slender bridges have appeared as material properties, design methods and building techniques have significantly improved. There has also been a strong community demand for more interesting structures, which are more aesthetic and appealing to the public. However, slender structures tend to be more sensitive to dynamic forces induced by wind loads $[4,5]$ or traffic flow [6], for instance, resulting in vibrations of the bridge deck. These vibrations can attain high magnitude in some cases, especially when the vortex-shedding frequency of the wind or the pace of the traffic approaches one of the natural frequencies of the bridge. A well-known example of failure caused by mechanical and aerodynamic effects is the collapse of the Tacoma Narrows Bridge [7]. Pedestrian-induced vibrations of slender footbridges have also been analysed by numerous authors. For a literature review of lateral vibrations see [6], while for vertical vibrations see for example [8] and the references therein. The most well-known example for dense pedestrian flow induced resonance of lateral vibration mode is the London Millennium Footbridge [9]. These examples have revealed that a proper dynamic analysis is necessary for slender bridges subjected to wind and traffic loads.

The application of some kind of suspension system for footbridge constructions is quite general. The disadvantage of cable suspension systems is that some cables can be highly overstressed while others can be slacked. High tension in cables is not desirable because it may lead to failure, but slacking of cables is also disadvantageous. Because cables do not have any resistance to compression, the dynamic behavior of suspended bridges can be highly nonlinear. Hence, a hanger system which offers a fairly uniform stress distribution in the cables has many advantages.

The present paper studies the dynamics of a beam hanged on a special suspension system, which is composed of pulleys and cables, and called the block-and-tackle suspension system. This effective suspension system was invented by Kolozsváry [10] for supporting tensile roofs $[11,12]$. It may also be used as a suspension system of footbridges, as suggested in [13], where a deck was suspended to a block-and-tackle suspension system and static analysis of the structure was accomplished. However, the dynamic behavior, which is very important in case of light and 


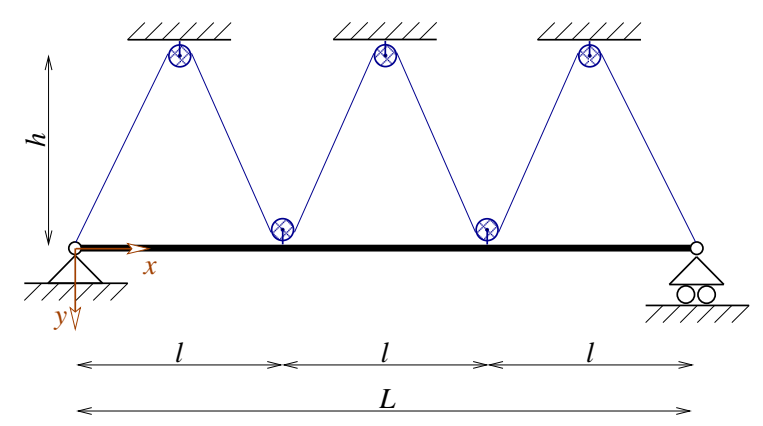

Figure 1: Model of a beam on a block-and-tackle suspension system

slender footbridges, has not been studied yet for such structures.

First the mechanical model is introduced in Section 2. Then in Section 3 the natural circular frequencies and the corresponding modal shape functions are derived for two states of the bridge. One state corresponds to slacked cables, and the other state corresponds to stressed cables. The obtained modal shape functions and frequencies are verified, and the modal decomposition based continuum approach for dynamic simulations is developed in Section 4. The vertical vibration of the structure due to vortex-shedding and passenger traffic is simulated and validated in Section 5. Finally, conclusions are drawn in Section 6.

\section{The mechanical model}

The mechanical model of the structure is shown in Figure 1. There is a simply supported Bernoulli-Euler beam of length $L$. Two pulleys are attached to this beam at equal distances. These pulleys divide the beam into three spans of length $\ell=L / 3$. The mass of the pulleys, and the friction between the pulleys and their shafts are neglected. There is a rigid upper structure at height $h$, to which three pulleys are attached, as shown in Figure 1. A massless, inextensible cable runs through the pulleys. The ends of the cable are fixed to the ends of the beam. The origin of a left-handed coordinate system is at the fixed support of the beam, the $x$-axis points to the right and coincides with the unloaded, straight axis of the beam, while the $y$-axis points downward. The loads act in the $x-y$ plane, and cause uniaxial bending about the $z$-axis. Lateral and lateral-torsional vibrations, and structural damping are neglected. Small displacements are assumed, $\hat{u}(x, t)$ denotes the vertical deflection of the beam.

\section{Natural circular frequencies and modal shape functions}

Let us introduce the dimensionless coordinate $\xi$ and time $\tau$ as:

$$
\begin{aligned}
& x=\xi L \quad \rightarrow \quad \frac{\partial \xi}{\partial x}=\frac{1}{L} \\
& t=\tau T \quad \rightarrow \quad \frac{\partial \tau}{\partial t}=\frac{1}{T} .
\end{aligned}
$$


Here the reference length $L=3 \ell$ is set to the total length of the beam and $T$ is a reference time period which will be fixed later, depending on the studied problem. The beam deflection can be given as function of the dimensionless variables:

$$
u(\xi, \tau)=\hat{u}(L \xi, T \tau) / L
$$

Then, partial differential equation that governs the free vibration of a Bernoulli-Euler beam [14] yields:

$$
\frac{E I}{L^{3}} \frac{\partial^{4} u(\xi, \tau)}{\partial \xi^{4}}+\frac{\mu L}{T^{2}} \frac{\partial^{2} u(\xi, \tau)}{\partial \tau^{2}}=0 .
$$

Here $E I$ is the bending stiffness of the beam and $\mu$ is its mass per unit length. The solution for (3) is searched for in the separated form

$$
u(\xi, \tau)=\sum_{i=1}^{\infty} u_{i}(\xi) \cdot\left(a_{i} \cos \left(2 \pi f_{i} T \tau\right)+b_{i} \sin \left(2 \pi f_{i} T \tau\right)\right)
$$

Here $f_{i}$ is the $i$ th natural frequency of the beam, while $u_{i}(\xi)$ is the corresponding dimensionless modal shape function. The coefficients $a_{i}$ and $b_{i}$ depend on initial conditions for prescribed shape functions. Substituting (4) in (3) leads to ordinary differential equations (ODEs):

$$
\frac{E I}{L^{3}} u_{i}^{I V}(\xi)-\left(2 \pi f_{i}\right)^{2} \mu L u_{i}(\xi)=0, \quad i=1,2, \ldots
$$

Here prime denotes differentiation with respect to $\xi$, hence $u_{i}^{I V}(\xi)=\mathrm{d}^{4} u_{i}(\xi) / \mathrm{d} \xi^{4}$.

Let us introduce the frequency parameter $r$ of the beam as:

$$
r=\frac{T}{L^{2}} \sqrt{\frac{E I}{\mu}} .
$$

Eq. (5) is solved by

$$
u_{i}(\xi)=A_{i} \cos \left(\lambda_{i} \xi\right)+B_{i} \sin \left(\lambda_{i} \xi\right)+C_{i} \cosh \left(\lambda_{i} \xi\right)+D_{i} \sinh \left(\lambda_{i} \xi\right),
$$

where

$$
\lambda_{i}=\sqrt{\frac{2 \pi f_{i} T}{r}}
$$

is the $i$ th eigenparameter, and the coefficients $A_{i}, B_{i}, C_{i}$, and $D_{i}$ are dependent on end conditions. Note that $u_{i}^{I V}(\xi)=\lambda_{i}^{4} u_{i}(\xi)$, and that $r \lambda_{i}^{2}$ is the $i$ th dimensionless natural circular frequency.

Since the suspension cable can bear only tension, it may introduce nonlinearity in the dynamics of the structure. This phenomenon is handled as follows. Two states of the structure are distinguished. If the cable is under tension, then the suspension system works as a load bearing structure. 
This is called the active state. If the tension in the cable vanishes, then the suspension system is passive, i.e. it does not play any load bearing role. This is called the passive state. Since the latter case is simpler, it is discussed first in Section 3.1, and then the active state is studied in Section 3.2.

\subsection{Passive suspension system}

In this case the studied structure is a simply supported beam of length $L$. The free vibration of such a beam is well known [14]. With our dimensionless notation the $i$ th eigenparameter and the corresponding modal shape function are:

$$
\begin{aligned}
& \lambda_{i}^{\mathrm{p}}=i \pi, \\
& u_{i}^{\mathrm{p}}(\xi)=\sqrt{2} \sin (i \pi \xi) .
\end{aligned}
$$

(Note that superscript "p" refers to the passive state.)

\subsection{Active suspension system}

In this case the cable is under tension and the suspension system contributes to the load-bearing capacity of the structure. Eq. (7) is valid for each span of length $\ell$. In order to compute the coefficients $A_{i}, B_{i}, C_{i}$, and $D_{i}$ of Eq. (7) for all the three spans, one needs to define $3 \times 4=12$ appropriate conditions. Four conditions come from the kinematic and kinetic boundary conditions at the beam ends, namely the pinned support and the roller do not allow vertical translations and cannot bear bending moments. Another four conditions come from the continuity of the beam at the suspended points in terms of displacements and slopes. Further two conditions prescribe zero jump in the bending moment at the suspended points. One condition arises from the inextensible property of the cable. The last one condition is obtained by relating the cable force to the jump in the shear force at the suspension points and eliminating the cable force. Since these conditions must be fulfilled at any time instant, the natural frequencies of the spans must be the same for a given modal shape. Hence, the boundary and continuity conditions are prescribed for the modal shape functions. Then, a frequency matrix can be compiled and analysed, leading to the eigenparameters and the corresponding modal shapes of the active state. This analysis is detailed in Appendix A. Each modal shape function is scaled so that it satisfies:

$$
\int_{0}^{1} u_{i}^{\mathrm{a}}(\xi) u_{i}^{\mathrm{a}}(\xi) \mathrm{d} \xi=1
$$

In general, the following identities hold for the modal shape functions:

$$
\int_{0}^{1} u_{i}(\xi) u_{j}(\xi) \mathrm{d} \xi=\delta_{i j}, \quad \int_{0}^{1} u_{i}^{I V}(\xi) u_{j}(\xi) \mathrm{d} \xi=\lambda_{i}^{4} \cdot \delta_{i j} .
$$

Here $\delta_{i j}$ is the Kronecker delta, i.e. $\delta_{i j}=1$ if $i=j$ and zero otherwise. The proof of Eq. (11) is given in Appendix A. In this paper only the first 12 active modes are used further. Figure 2 shows 


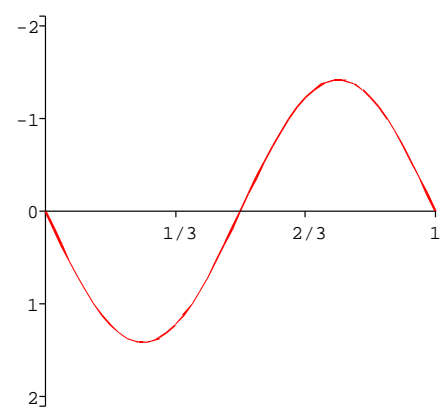

Mode $1, \lambda_{1}^{\mathrm{a}}=2 \pi$

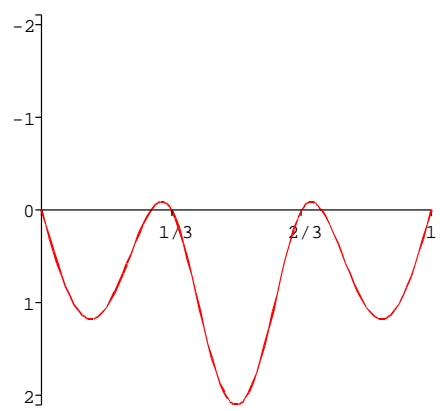

Mode $4, \lambda_{4}^{\mathrm{a}}=4.1038385 \pi$

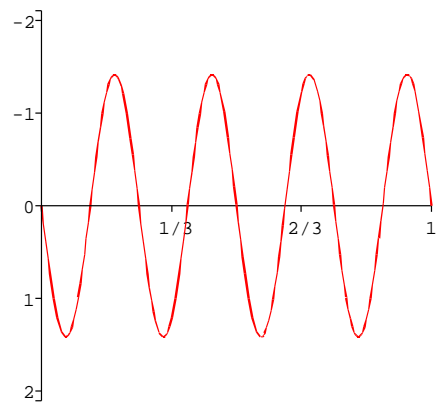

Mode $7, \lambda_{7}^{\mathrm{a}}=8 \pi$

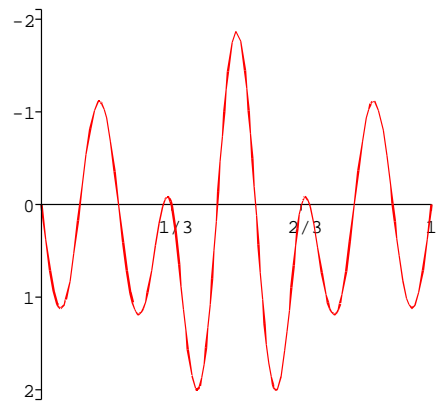

Mode $10, \lambda_{10}^{\mathrm{a}}=10.095097 \pi$

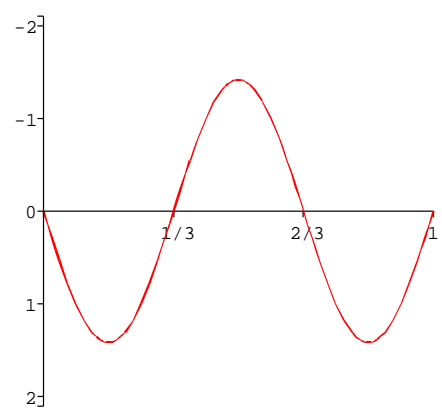

Mode $2, \lambda_{2}^{\mathrm{a}}=3 \pi$

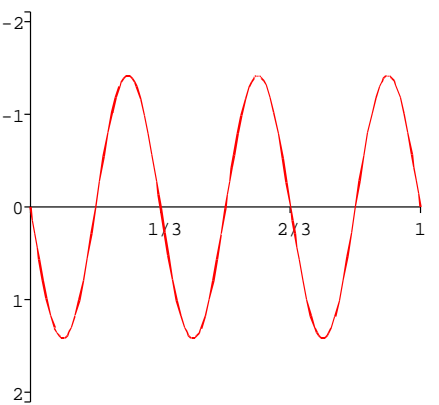

Mode $5, \lambda_{5}^{\mathrm{a}}=6 \pi$

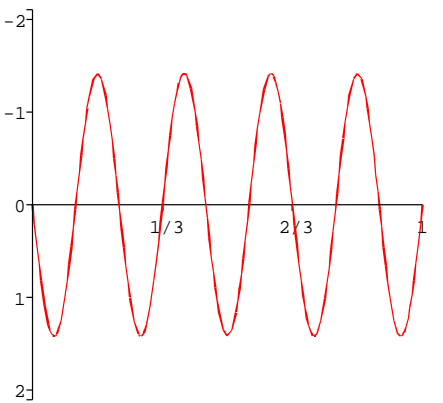

Mode $8, \lambda_{8}^{\mathrm{a}}=9 \pi$

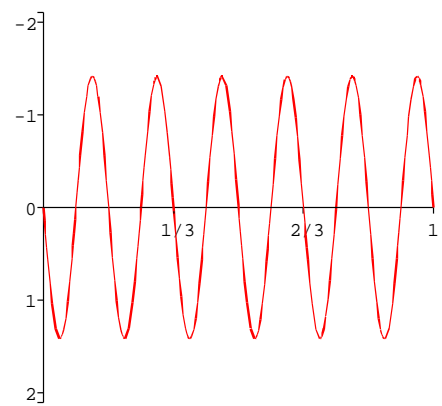

Mode $11, \lambda_{11}^{\mathrm{a}}=12 \pi$

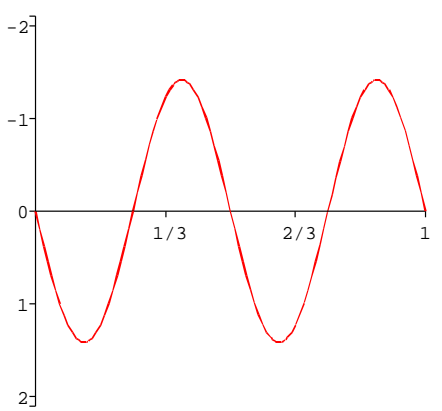

Mode $3, \lambda_{3}^{\mathrm{a}}=4 \pi$

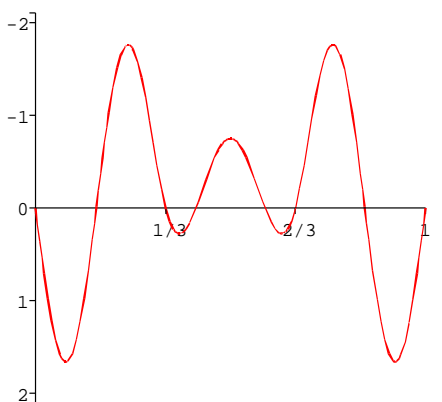

Mode $6, \lambda_{6}^{\mathrm{a}}=6.4052817 \pi$

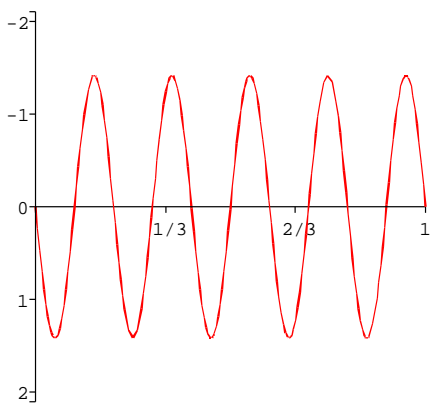

Mode $9, \lambda_{9}^{\mathrm{a}}=10 \pi$

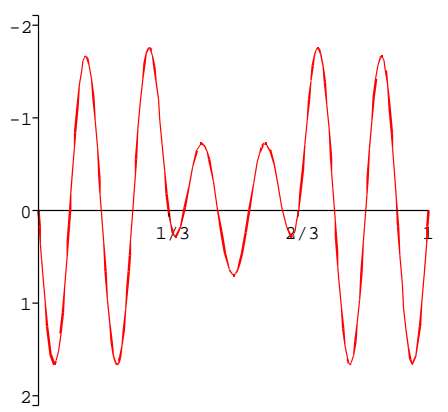

Mode $12, \lambda_{12}^{\mathrm{a}}=12.404920 \pi$

Figure 2: The first twelve normalized modal shape functions of the active state and the corresponding eigenparameters. Note that the natural frequency can be computed as: $f_{i}^{\mathrm{a}}=\frac{1}{2 \pi} \frac{r \cdot\left(\lambda_{i}^{\mathrm{a}}\right)^{2}}{T}=\frac{1}{2 \pi} \frac{\left(\lambda_{i}^{\mathrm{a}}\right)^{2}}{L^{2}} \sqrt{\frac{E I}{\mu}}$ for a given beam. 
these first 12 modal shape functions of the active state normalized according to (10). Is it worth noting that odd shape functions are skew-symmetric, while even shape functions are symmetric. Classification of the eigenparameters and the corresponding eigenmodes are thoroughly discussed in Appendix A. Below only the main results, i.e. the trivial and non-trivial active modes are presented.

\subsubsection{Trivial modes of the active state}

If $\bmod (i, 6)$ equals $1,2,3$ or 5 , then the $i$ th eigenparameter and the corresponding modal shape are:

$$
\begin{aligned}
& \lambda_{i}^{\mathrm{a}}=(i+1) \pi, \\
& u_{i}^{\mathrm{a}}=\sqrt{2} \sin ((i+1) \pi \xi),
\end{aligned}
$$

while the cable force is zero. Such a modal shape function is called trivial because it coincides with the $(i+1)$ st modal shape function of the passive state (see Eq. (9)). It has either a skewsymmetric form (for odd $i$ values) or nodes at the suspended points (for those $i$ values that satisfy $\bmod (i, 6)=2$ or 5 , see the mid column of Figure 2$)$. For example, modes 1 and 3 are skewsymmetric, mode 2 is symmetric but has nodes at the suspended points, while mode 5 fulfills both conditions.

\subsubsection{Non-trivial modes of the active state}

If $\bmod (i, 6)$ equals 0 or 4 , then the following inequalities hold for the $i$ th eigenparameter:

$$
i \pi<\lambda_{i}^{\mathrm{a}}<(i+1) \pi
$$

The corresponding cable force is non-zero and the modal shape function is symmetric. The first 4 non-trivial shape functions of the active state are given in Appendix A.

\section{Non-linear vibrations of the bilinear structure: theory}

As long as the state of the suspension system is unchanged, the structure behaves as a (linear elastic) Bernoulli-Euler beam, and undergoes linear vibrations under harmonic loadings. But our structure has different stiffness if the suspension system is active than if it is passive, hence it is bilinear. Therefore, changes in the state of the suspension system can make the vibration of the structure non-linear, in spite of there are linear vibrations between two consecutive state changes.

It is well known that bilinear oscillators may exhibit periodic, quasi-periodic, or chaotic motions [15]. A relevant example is the beam with non-linear boundary conditions [16], which was reduced to a single-degree-of-freedom system and shown to possess chaotic vibrations.

In the followings, it is shown how the linear system is analysed, and the active shape functions are verified with a static loading. Then a procedure is developed for handling the state changes and simulating vibrations of the bilinear structure. 


\subsection{General remarks on the vibrations in a fixed state}

The partial differential equation of the forced vibration of a Bernoulli-Euler beam [14] with dimensionless spatial and temporal variables (1) is

$$
\frac{E I}{L^{3}} \frac{\partial^{4} u(\xi, \tau)}{\partial \xi^{4}}+\frac{\mu L}{T^{2}} \frac{\partial^{2} u(\xi, \tau)}{\partial \tau^{2}}=q(\xi, \tau)
$$

Here $q(\xi, \tau)=\hat{q}(L \xi, T \tau)$, with $\hat{q}(x, t)$ being a time-dependent distributed load. The complete solution for (12) is the sum of the homogeneous and the particular solutions. The homogeneous solution is given by (4). The particular solution is searched for as a time-dependent combination of the modal shape functions:

$$
u_{q}(\xi, \tau)=\sum_{j=1}^{\infty} u_{j}(\xi) \cdot \eta_{j}(\tau) .
$$

In both (4) and (13) those modal shape functions are used that correspond to the actual state of the suspension system (active or passive). A change in the state of the suspension system introduces nonlinearity to the problem. It is explained later how this nonlinearity is handled. Now the time interval while the suspension system is either active or passive is studied.

Eq. (13) is substituted in (12), then the result is multiplied by $u_{i}(\xi)$ and integrated with respect to $\xi$ on $[0,1]$. Using (11) it yields the following equality:

$$
\frac{E I}{L^{3}} \lambda_{i}^{4} \eta_{i}(\tau)+\frac{\mu L}{T^{2}} \ddot{\eta}_{i}(\tau)=\int_{0}^{1} q(\xi, \tau) u_{i}(\xi) \mathrm{d} \xi
$$

Here dot denotes differentiation with respect to $\tau$. Now let us multiply the above equality by $T^{2} /(\mu L)$, implement the frequency parameter (6), and introduce the $i$ th modal forcing:

$$
Q_{i}(\tau)=\frac{T^{2}}{\mu L} \int_{0}^{1} q(\xi, \tau) u_{i}(\xi) \mathrm{d} \xi .
$$

Thus, the following system of ODEs is developed:

$$
\ddot{\eta}_{i}(\tau)+\left(r \lambda_{i}^{2}\right)^{2} \eta_{i}(\tau)=Q_{i}(\tau), \quad i=1,2, \ldots
$$

The solutions for (15) yield the dimensionless modal displacements, i.e. the time-dependent functions in the combination (13), and so the particular solution of the forced vibration can also be generated. 


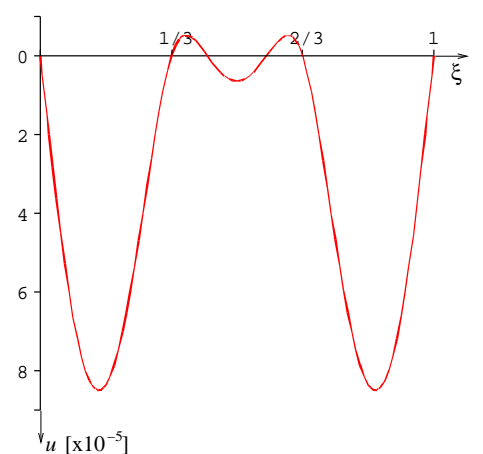

(a)

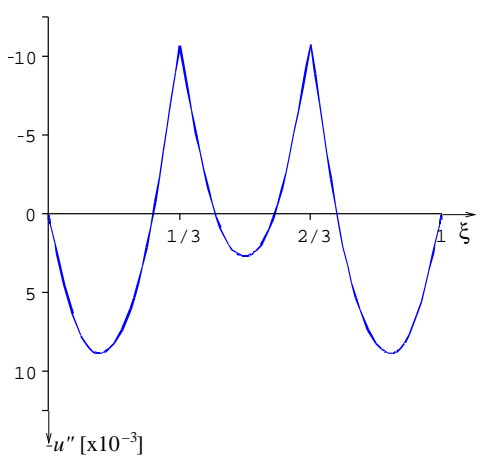

(b)

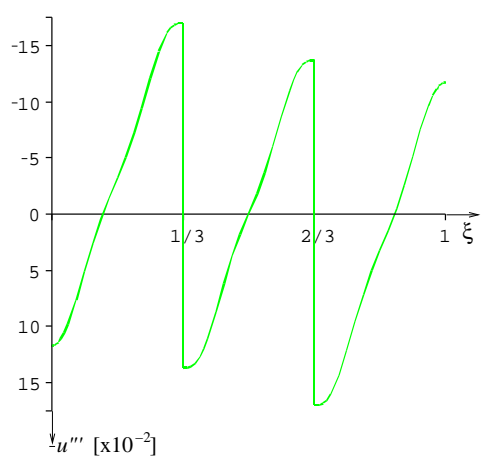

(c)

Figure 3: (a) Approximate static deflection of the beam, $u(\xi)$, under a constant distributed load using twelve modal shapes of the active state. (b) The curvature, $-u^{\prime \prime}$, which is proportional to the bending moment diagram of the beam. (c) Opposite of the third derivative of the deflection, $-u^{\prime \prime \prime}$, which is proportional to the shear force diagram of the beam. $q_{\mathrm{s}} T^{2} /\left(\mu L r^{2}\right)=1$. Note that the exact shear force diagram would be piece-wise linear.

\subsection{A static distributed load: verifying the modal shapes of the active state}

First the case of a constant, static distributed load of magnitude $q_{\mathrm{s}}$ is studied. The initial conditions are set such that $a_{i}$ and $b_{i}$ in (4) are zero. The modal force (14) is time-independent:

$$
Q_{i}^{\mathrm{s}}=\frac{q_{\mathrm{s}} T^{2}}{\mu L} \int_{0}^{1} u_{i}(\xi) \mathrm{d} \xi=\frac{q_{\mathrm{s}} T^{2}}{\mu L} F_{i}
$$

Here $F_{i}$ is the definite integral (area) of the $i$ th modal shape function. Therefore, the modal force is zero if the corresponding modal shape function is skew-symmetric. The solution for (15) is also time-independent:

$$
\eta_{i}^{\mathrm{s}}=\frac{q_{\mathrm{s}} T^{2}}{\mu L r^{2}} \cdot \frac{F_{i}}{\lambda_{i}^{4}}
$$

If the load points downward, i.e. $q_{\mathrm{s}}>0$, then the state is active, hence in (17) the active modal shape functions and eigenparameters have to be used. Figure 3 (a) shows the deflection $u(\xi)$ of the beam for $q_{\mathrm{s}} T^{2} /\left(\mu L r^{2}\right)=1$, using 12 modal shape functions of the active state. Figure 3 (b) and (c) show the opposite of the second and third derivatives of $u(\xi)$, which are proportional to the bending moment and shear force diagrams, respectively. Note that the exact shear force diagram is piece-wise linear, and Figure 3 (c) is its best approximation with 12 modal shapes.

\subsubsection{Verification for bending moment}

For the constant distributed loading the static behavior of the beam suspended to an inextensible cable is identical to that of the continuous three-span beam. Hence, our results can be compared to the known, analytic solution of a three-span beam subjected to uniformly distributed load ([17], p. 349). A good agreement between the two solutions would verify the modal shape functions and 
natural frequencies of the active state. According to [17], the bending moment at a supported mid point is:

$$
M(L / 3)=-\frac{q_{\mathrm{s}} L^{2}}{90}
$$

It yields the dimensionless curvature

$$
\kappa(1 / 3)=-u^{\prime \prime}(1 / 3)=L \frac{M}{E I}=-\frac{1}{90} \cdot \frac{q_{\mathrm{s}} T^{2}}{\mu L r^{2}} .
$$

For $q_{\mathrm{s}} T^{2} /\left(\mu L r^{2}\right)=1$, the computed curvature is -0.01076 with 12 modal shapes of the active state. The exact result is $-1 / 90$, hence the relative error yields $3.171 \%$. (Note that this relative error is independent of the value of $q_{\mathrm{s}} T^{2} /\left(\mu L r^{2}\right)$.) This acceptable error arises from the finitization of the function space: only a finite number of modal shapes have been taken into account.

\subsubsection{A worked example for displacements}

A worked example is shown now for the computation of the real values of the displacements, using the verified, dimensionless modal shapes of the active (suspended) state. A beam of bending stiffness $E I=0.3 \mathrm{MNm}^{2}$ and length $L=18 \mathrm{~m}$ is subjected to a constant distributed load $q_{\mathrm{p}}=$ $750 \mathrm{~N} / \mathrm{m}$.

From Figure 3 (a) the dimensionless displacement of the mid point of the beam is $u_{\text {mid }}=$ $0.6393 \cdot 10^{-5}$. It belongs to $q_{\mathrm{s}} T^{2} /\left(\mu L r^{2}\right)=1$, which, in view of Eq. (6), yields:

$$
q_{\mathrm{s}}=\frac{\mu L r^{2}}{T^{2}}=\frac{E I}{L^{3}}=51.44 \mathrm{~N} / \mathrm{m}
$$

Finally, the dimensionless displacement $u_{\text {mid }}$ is rescaled to the load $q_{\mathrm{p}}$, and its real value is computed based on Eq. (2):

$$
\hat{u}_{\text {mid }}=u_{\text {mid }} \frac{q_{\mathrm{p}}}{q_{\mathrm{s}}} L=1.678 \cdot 10^{-3} \mathrm{~m} .
$$

\subsection{A distributed load with both static and dynamic components}

The beam is under a static distributed load of magnitude $q_{\mathrm{s}}$, and a dynamic distributed load of amplitude $q_{0}$ varying harmonically in time with frequency $f$. The ratio of the static load to the dynamic amplitude is denoted by $\alpha$ :

$$
q_{\mathrm{s}}=\alpha q_{0} .
$$

The distributed load is then $\hat{q}(x, t)=q_{0}(\alpha+\sin (2 \pi f t))$. The unit of time $T$ is set equal to the reciprocal of the frequency $f$. The forcing with dimensionless variables is

$$
q(\xi, \tau)=q_{0}(\alpha+\sin (2 \pi \tau))
$$


hence the modal force (14) has the form of

$$
Q_{i}^{\mathrm{h}}(\tau)=\frac{q_{0} T^{2}}{\mu L}(\alpha+\sin (2 \pi \tau)) \int_{0}^{1} u_{i}(\xi) \mathrm{d} \xi=\frac{q_{0} T^{2}}{\mu L} F_{i}(\alpha+\sin (2 \pi \tau)) .
$$

The modal force is zero if the corresponding modal shape function is odd. Now (15) yields

$$
\ddot{\eta}_{i}(\tau)+\left(r \lambda_{i}^{2}\right)^{2} \eta_{i}(\tau)=\frac{q_{0} T^{2}}{\mu L} F_{i}(\alpha+\sin (2 \pi \tau)),
$$

which is essentially the ODE of a spring-mass system subjected to a static and a harmonic forcing. The complete solution for the modal displacement $\eta_{i}(\tau)$ is the sum of the time-dependent part of the free vibration (4) and the particular solution of (20):

$$
\eta_{i}(\tau)=a_{i} \cos \left(r \lambda_{i}^{2} \tau\right)+b_{i} \sin \left(r \lambda_{i}^{2} \tau\right)+\frac{q_{0} T^{2}}{\mu L r^{2}} \cdot \frac{F_{i}}{\lambda_{i}^{4}} \cdot\left(\alpha+\frac{1}{1-\left(\frac{2 \pi}{r \lambda_{i}^{2}}\right)^{2}} \sin (2 \pi \tau)\right) .
$$

Here $a_{i}$ and $b_{i}$ are from initial conditions. Note that if the dimension of $\mu$ is properly chosen, then only two parameters are needed: the frequency parameter $r$, and the ratio of the static to dynamic load $\alpha$.

The $i$ th dimensionless natural period of vibration in the units of the reference period $T$ is:

$$
T_{\mathrm{n}, i}=\frac{2 \pi}{r \lambda_{i}^{2}}
$$

It is also worth mentioning that the period of harmonic forcing is always one in the units of $T$.

If a finite number $N$ of modal shape functions are used, then the modal displacements, $\boldsymbol{\eta}=$ $\left[\eta_{1}(\tau), \ldots, \eta_{N}(\tau)\right]^{T}$, and the modal velocities, $\dot{\boldsymbol{\eta}}=\left[\dot{\eta}_{1}(\tau), \ldots, \dot{\eta}_{N}(\tau)\right]^{T}$, can be written as:

$$
\begin{aligned}
& \boldsymbol{\eta}(\tau)=\mathbf{C}(\tau) \mathbf{a}+\mathbf{S}(\tau) \mathbf{b}+\alpha \mathbf{h}+\sin (2 \pi \tau) \mathbf{R}_{\mathrm{d}} \mathbf{h}, \\
& \dot{\boldsymbol{\eta}}(\tau)=\boldsymbol{\Lambda}(-\mathbf{S}(\tau) \mathbf{a}+\mathbf{C}(\tau) \mathbf{b})+2 \pi \cos (2 \pi \tau) \mathbf{R}_{\mathrm{d}} \mathbf{h}
\end{aligned}
$$


Here

$$
\begin{aligned}
\mathbf{C}(\tau) & =\left\langle\cos \left(r \lambda_{1}^{2} \tau\right), \cos \left(r \lambda_{2}^{2} \tau\right), \ldots, \cos \left(r \lambda_{N}^{2} \tau\right)\right\rangle, \\
\mathbf{S}(\tau) & =\left\langle\sin \left(r \lambda_{1}^{2} \tau\right), \sin \left(r \lambda_{2}^{2} \tau\right), \ldots, \sin \left(r \lambda_{N}^{2} \tau\right)\right\rangle, \\
\boldsymbol{\Lambda} & =r\left\langle\lambda_{1}^{2}, \lambda_{2}^{2}, \ldots, \lambda_{N}^{2}\right\rangle, \\
\mathbf{R}_{\mathrm{d}} & =\left\langle\frac{1}{1-\left(\frac{2 \pi}{r \lambda_{1}^{2}}\right)^{2}}, \frac{1}{1-\left(\frac{2 \pi}{r \lambda_{2}^{2}}\right)^{2}}, \ldots, \frac{1}{1-\left(\frac{2 \pi}{r \lambda_{N}^{2}}\right)^{2}}\right\rangle
\end{aligned}
$$

are $N$-by- $N$ diagonal matrices, and

$$
\mathbf{a}=\left[a_{1}, a_{2}, \ldots, a_{N}\right]^{T}, \quad \mathbf{b}=\left[b_{1}, b_{2}, \ldots, b_{N}\right]^{T}
$$

are $N$-vectors that depend on initial conditions, while the load vector is:

$$
\mathbf{h}=\frac{q_{0} T^{2}}{\mu L r^{2}}\left[\frac{F_{1}}{\lambda_{1}^{4}}, \frac{F_{2}}{\lambda_{2}^{4}}, \ldots, \frac{F_{N}}{\lambda_{N}^{4}}\right]^{T} .
$$

Note that entry $i, i$ of $\mathbf{R}_{\mathrm{d}}$, denoted by $R_{\mathrm{d}, i}$, is the deformation response factor for the $i$ th mode.

If $\boldsymbol{\eta}$ and $\dot{\boldsymbol{\eta}}$ are given at some time instant $\tau$, then a and b can be computed from (23) and (24):

$$
\begin{aligned}
& \mathbf{a}=\mathbf{C}\left(\boldsymbol{\eta}-\alpha \mathbf{h}-\sin (2 \pi \tau) \mathbf{R}_{\mathrm{d}} \mathbf{h}\right)-\boldsymbol{\Lambda}^{-1} \mathbf{S}\left(\dot{\boldsymbol{\eta}}-2 \pi \cos (2 \pi \tau) \mathbf{R}_{\mathrm{d}} \mathbf{h}\right), \\
& \mathbf{b}=\mathbf{S}\left(\boldsymbol{\eta}-\alpha \mathbf{h}-\sin (2 \pi \tau) \mathbf{R}_{\mathrm{d}} \mathbf{h}\right)+\boldsymbol{\Lambda}^{-1} \mathbf{C}\left(\dot{\boldsymbol{\eta}}-2 \pi \cos (2 \pi \tau) \mathbf{R}_{\mathrm{d}} \mathbf{h}\right) .
\end{aligned}
$$

Equations (23)-(27) hold for both active and passive states, only the corresponding (active or passive) eigenparameters $\lambda_{i}$ and modal shapes $u_{i}(\xi)$ are needed for a fixed number of modes.

\subsection{Nonlinearity induced by activating and inactivating the suspension system}

\subsubsection{Transformation between active and passive states}

The displacement of the beam is a linear combination of the modal shape functions. If the suspension system is active, then the displacement is:

$$
u(\xi, \tau)=\sum_{i=1}^{\infty} u_{i}^{\mathrm{a}}(\xi) \eta_{i}^{\mathrm{a}}(\tau)=\left(\mathbf{u}_{\infty}^{\mathrm{a}}\right)^{T} \boldsymbol{\eta}_{\infty}^{\mathrm{a}} .
$$

Here the vector of infinite depth $\mathbf{u}_{\infty}^{\mathrm{a}}=\left[u_{1}^{\mathrm{a}}(\xi), u_{2}^{\mathrm{a}}(\xi), \ldots, u_{\infty}^{\mathrm{a}}(\xi)\right]^{T}$ collects the modal shape functions of the active state, and $\boldsymbol{\eta}_{\infty}^{\mathrm{a}}=\left[\eta_{1}^{\mathrm{a}}(\tau), \eta_{2}^{\mathrm{a}}(\tau), \ldots, \eta_{\infty}^{\mathrm{a}}(\tau)\right]^{T}$ stores the corresponding timedependent functions. If the suspension system is passive, then the displacement function is

$$
u(\xi, \tau)=\sum_{i=1}^{\infty} u_{i}^{\mathrm{p}}(\xi) \eta_{i}^{\mathrm{p}}(\tau)=\left(\mathbf{u}_{\infty}^{\mathrm{p}}\right)^{T} \boldsymbol{\eta}_{\infty}^{\mathrm{p}} .
$$


At a transition, i.e. at a time instant when the suspension system is activated or inactivated, the deflection of the beam can be described by the modal shapes of both states, i.e.

$$
\left(\mathbf{u}_{\infty}^{\mathrm{p}}\right)^{T} \boldsymbol{\eta}_{\infty, \mathrm{tr}}^{\mathrm{p}}=\left(\mathbf{u}_{\infty}^{\mathrm{a}}\right)^{T} \boldsymbol{\eta}_{\infty, \mathrm{tr}}^{\mathrm{a}} .
$$

Multiplying the above formula by $\mathbf{u}_{\infty}^{\mathrm{p}}$ from the left and integrating it with respect to $\xi$ over $[0,1]$ yields:

$$
\boldsymbol{\eta}_{\infty, \mathrm{tr}}^{\mathrm{p}}=\mathbf{T}_{\infty} \boldsymbol{\eta}_{\infty, \mathrm{tr}}^{\mathrm{a}}
$$

Here the time-independent transformation matrix $\mathbf{T}_{\infty}$ is the following definite integral:

$$
\mathbf{T}_{\infty}=\int_{0}^{1} \mathbf{u}_{\infty}^{\mathrm{p}}\left(\mathbf{u}_{\infty}^{\mathrm{a}}\right)^{T} \mathrm{~d} \xi
$$

Similarly, if (28) is multiplied by $\mathbf{u}_{\infty}^{\mathrm{a}}$ from the left and integrated with respect to $\xi$ over $[0,1]$, then the inverse transformation is obtained:

$$
\boldsymbol{\eta}_{\infty, \mathrm{tr}}^{\mathrm{a}}=\mathbf{T}_{\infty}^{T} \boldsymbol{\eta}_{\infty, \mathrm{tr}}^{\mathrm{p}}
$$

From (29) and (30) it can be seen that $\mathbf{T}_{\infty}^{T} \mathbf{T}_{\infty}=\mathbf{T}_{\infty} \mathbf{T}_{\infty}^{T}=\mathbf{I}_{\infty}$ is the (infinite-by-infinite) identity matrix.

The dimensionless mechanical energy, associated with the $i$ th modal shape, is:

$$
\psi_{i}=\frac{1}{2}\left(\dot{\eta}_{i}\right)^{2}+\frac{r^{2} \lambda_{i}^{4}}{2}\left(\eta_{i}\right)^{2}
$$

At a transition, when the suspension system is inactivated, the mechanical energy of an active mode should be all transfered to the corresponding passive mode(s). Using Eq. (29) the conservation of the total mechanical energy can be formulated at the transition:

$$
\begin{aligned}
\psi_{\text {tot }} & =\frac{1}{2}\left(\dot{\boldsymbol{\eta}}_{\infty, \mathrm{tr}}^{\mathrm{a}}\right)^{T} \dot{\boldsymbol{\eta}}_{\infty, \mathrm{tr}}^{\mathrm{a}}+\frac{1}{2}\left(\boldsymbol{\eta}_{\infty, \mathrm{tr}}^{\mathrm{a}}\right)^{T}\left(\boldsymbol{\Lambda}^{\mathrm{a}}\right)^{2} \boldsymbol{\eta}_{\infty, \mathrm{tr}}^{\mathrm{a}}=\frac{1}{2}\left(\dot{\boldsymbol{\eta}}_{\infty, \mathrm{tr}}^{\mathrm{p}}\right)^{T} \dot{\boldsymbol{\eta}}_{\infty, \mathrm{tr}}^{\mathrm{p}}+\frac{1}{2}\left(\boldsymbol{\eta}_{\infty, \mathrm{tr}}^{\mathrm{p}}\right)^{T}\left(\boldsymbol{\Lambda}^{\mathrm{p}}\right)^{2} \boldsymbol{\eta}_{\infty, \mathrm{tr}}^{\mathrm{p}} \\
& =\frac{1}{2}\left(\dot{\boldsymbol{\eta}}_{\infty, \mathrm{tr}}^{\mathrm{a}}\right)^{T} \mathbf{T}_{\infty}^{T} \mathbf{T}_{\infty} \dot{\boldsymbol{\eta}}_{\infty, \mathrm{tr}}^{\mathrm{a}}+\frac{1}{2}\left(\boldsymbol{\eta}_{\infty, \mathrm{tr}}^{\mathrm{a}}\right)^{T} \mathbf{T}_{\infty}^{T}\left(\boldsymbol{\Lambda}^{\mathrm{p}}\right)^{2} \mathbf{T}_{\infty} \boldsymbol{\eta}_{\infty, \mathrm{tr}}^{\mathrm{a}} .
\end{aligned}
$$

Since $\mathbf{T}_{\infty}^{T} \mathbf{T}_{\infty}=\mathbf{I}_{\infty}$, the mechanical energy is preserved, there is no energy input or dissipation during a state change of the suspension system.

However, in numerical analysis it is not possible to use all the infinitely many modal shape functions. Only a finite number $N^{\mathrm{a}}$ of active modal shapes and $N^{\mathrm{p}}$ of passive modal shapes can be implemented, which leads to an $N^{\mathrm{p}}-$ by $-N^{\mathrm{a}}$ matrix $\mathbf{T}$. In that case, the $N^{\mathrm{a}}-$ by $-N^{\mathrm{a}}$ matrix $\mathbf{T}^{T} \mathbf{T}$ differs from the unit matrix and some mechanical energy dissipates from the system each time the suspension system is activated (and so when it is inactivated). The closest $\mathbf{T}^{T} \mathbf{T}$ is to the unit matrix (i.e. the closest the eigenvalues of $\mathbf{T}^{T} \mathbf{T}$ are to one), the less energy loss occurs during a 
transition. The optimal numbers of active and passive modes are $N$ and $N+1$, respectively, since the $i$ th trivial active mode corresponds to the $(i+1)$ st passive mode. In our numerical examples $N^{\mathrm{a}}=12$ and $N^{\mathrm{p}}=13$ modal shapes are used, leading to the $N^{\mathrm{p}}-$ by $-N^{\mathrm{a}}$ transformation matrix:

$$
\mathbf{T}=\left[\begin{array}{cccccccccccc}
0 & 0 & 0 & 0.7661 & 0 & -0.3326 & 0 & 0 & 0 & 0.3141 & 0 & -0.1717 \\
\mathbf{1} & 0 & 0 & 0 & 0 & 0 & 0 & 0 & 0 & 0 & 0 & 0 \\
0 & \mathbf{1} & 0 & 0 & 0 & 0 & 0 & 0 & 0 & 0 & 0 & 0 \\
0 & 0 & \mathbf{1} & 0 & 0 & 0 & 0 & 0 & 0 & 0 & 0 & 0 \\
0 & 0 & 0 & 0.6343 & 0 & 0.5286 & 0 & 0 & 0 & -0.3342 & 0 & 0.1764 \\
0 & 0 & 0 & 0 & \mathbf{1} & 0 & 0 & 0 & 0 & 0 & 0 & 0 \\
0 & 0 & 0 & -0.1023 & 0 & 0.7795 & 0 & 0 & 0 & 0.4085 & 0 & -0.1911 \\
0 & 0 & 0 & 0 & 0 & 0 & \mathbf{1} & 0 & 0 & 0 & 0 & 0 \\
0 & 0 & 0 & 0 & 0 & 0 & 0 & \mathbf{1} & 0 & 0 & 0 & 0 \\
0 & 0 & 0 & 0 & 0 & 0 & 0 & 0 & \mathbf{1} & 0 & 0 & 0 \\
0 & 0 & 0 & 0.01508 & 0 & -0.04317 & 0 & 0 & 0 & 0.7666 & 0 & 0.4499 \\
0 & 0 & 0 & 0 & 0 & 0 & 0 & 0 & 0 & 0 & \mathbf{1} & 0 \\
0 & 0 & 0 & -0.007657 & 0 & 0.02081 & 0 & 0 & 0 & -0.1795 & 0 & 0.8330
\end{array}\right] .
$$

Note that columns 4, 6, 10, and 12, that correspond to non-trivial active modes, are not unit vectors.

Although the conservation of the total mechanical energy is violated by using a finite number of modes, it can be thought of as a contribution of the suspension system to the dynamics of the structure. The cable has not only kinematic and static effects (taken into account in the modal shapes and frequencies), but it dissipates some energy while activated or inactivated.

\subsubsection{Transition conditions}

The jump in the dimensionless shear force diagram at the left suspension point,

$$
\text { jump }=u^{\prime \prime \prime}(1 / 3+\varepsilon)-u^{\prime \prime \prime}(1 / 3-\varepsilon),
$$

is proportional to the cable force. (Here $\varepsilon$ is a sufficiently small number.) If the structure is in the active state and the sign of the cable force turns from positive to negative, then the suspension system becomes passive.

The inextensibility condition is:

$$
\text { inext }=u(1 / 3)+u(2 / 3) .
$$

If the structure is in the passive state and the above value turns from negative to positive, then the suspension system becomes active.

\subsubsection{Switching between active and passive states}

The procedure of the numerical simulation is as follows. It is assumed that in the beginning of the simulation the structure is unloaded and the suspension cable is neither stressed nor slacked. The simulation starts with a chosen state, the active state in this case. First initial conditions are set. For simplicity $\mathbf{a}^{\mathbf{a}}=\mathbf{b}^{\mathbf{a}}=\mathbf{0}$ are prescribed. A sufficiently small time step $\Delta \tau$ is also set. 
Starting at $\tau=0$ the dimensionless time $\tau$ is increased by small steps $\Delta \tau$ and $\eta^{\mathrm{a}}(\tau)$ is computed from (23) at each step. There, eigenparameters $\lambda_{i}^{\mathrm{a}}$ and modal shapes $u_{i}^{\mathrm{a}}(\xi)$ of the active state are used. After each time step it is checked whether the cable force has become compressed or not. It can be done by evaluating (32). The cable has become compressed between steps $k-1$ and $k$ if jump $_{k-1}>0$ and jump ${ }_{k}<0$. If so then a time step smaller than $\Delta \tau$ is used after the $(k-1)$ st step. This time step is approximated by $\beta^{\mathrm{p}} \Delta \tau$, where $\beta^{\mathrm{p}}$ is from piece-wise linearization:

$$
\beta^{\mathrm{p}}=\frac{\text { jump }_{k-1}}{\text { jump }_{k-1}-\text { jump }_{k}} .
$$

At the time instant of transition, $\tau_{\mathrm{tr}}=\left(k-1+\beta^{\mathrm{p}}\right) \Delta \tau$, both $\boldsymbol{\eta}_{\mathrm{tr}}^{\mathrm{a}}$ and $\dot{\boldsymbol{\eta}}_{\mathrm{tr}}^{\mathrm{a}}$ are computed from (23) and (24). Then a switch between active and passive states is accomplished by using the transformation (29), which holds for both the displacements and the velocities:

$$
\boldsymbol{\eta}_{\mathrm{tr}}^{\mathrm{p}}=\mathbf{T} \boldsymbol{\eta}_{\mathrm{tr}}^{\mathrm{a}}, \quad \dot{\boldsymbol{\eta}}_{\mathrm{tr}}^{\mathrm{p}}=\mathbf{T} \dot{\boldsymbol{\eta}}_{\mathrm{tr}}^{\mathrm{a}}
$$

Before continuing the simulation with the passive state of the suspension system, the actual initial conditions $\mathbf{a}^{\mathrm{p}}$ and $\mathbf{b}^{\mathrm{p}}$ are computed from (27). There the previously determined $\boldsymbol{\eta}_{\mathrm{tr}}^{\mathrm{p}}$ and $\dot{\eta}_{\mathrm{tr}}^{\mathrm{p}}$ are used with the eigenparameters $\lambda_{i}^{\mathrm{p}}$ and modal shapes $u_{i}^{\mathrm{p}}(\xi)$ of the passive state. Then the numerical integration is continued and the values of $\boldsymbol{\eta}^{\mathrm{p}}$ are computed at each time step from (23). The length of the first step is set to $\left(1-\beta^{\mathrm{p}}\right) \Delta \tau$ in order to have the solution in equidistant time steps. After each iteration, it must be checked whether the suspension system becomes active or not by evaluating (33). The cable should have been activated and the suspension system should have already been bearing loads somewhere between steps $m-1$ and $m$ if inext ${ }_{m-1}<0$ and inext $_{m}>0$. In that case a smaller time step, denoted by $\beta^{\mathrm{a}} \Delta \tau$, is needed, where

$$
\beta^{\mathrm{a}}=\frac{\text { inext }_{m-1}}{\text { inext }_{m-1}-\text { inext }_{m}} \text {. }
$$

The modal displacements and velocities $\boldsymbol{\eta}_{\mathrm{tr}}^{\mathrm{p}}$ and $\dot{\boldsymbol{\eta}}_{\mathrm{tr}}^{\mathrm{p}}$ are computed at this time instant of transition, $\tau_{\text {tr }}=\left(m-1+\beta^{\mathrm{a}}\right) \Delta \tau$. Then the suspension system is activated by using the inverse transformation (30) for both the displacements and the velocities:

$$
\boldsymbol{\eta}_{\mathrm{tr}}^{\mathrm{a}}=\mathbf{T}^{T} \boldsymbol{\eta}_{\mathrm{tr}}^{\mathrm{p}}, \quad \dot{\boldsymbol{\eta}}_{\mathrm{tr}}^{\mathrm{a}}=\mathbf{T}^{T} \dot{\boldsymbol{\eta}}_{\mathrm{tr}}^{\mathrm{p}}
$$

Integrating the system continues then with the active modes using Eq. (23). The initial values $\mathrm{a}^{\mathrm{a}}$ and $\mathrm{b}^{\mathrm{a}}$ are computed from (27), using the freshly determined $\boldsymbol{\eta}_{\mathrm{tr}}^{\mathrm{a}}$ and $\dot{\boldsymbol{\eta}}_{\mathrm{tr}}^{\mathrm{a}}$, along with the eigenparameters $\lambda_{i}^{\mathrm{a}}$ and modal shapes $u_{i}^{\mathrm{a}}(\xi)$ of the active state. The length of the first time step is only $\left(1-\beta^{\mathrm{a}}\right) \Delta \tau$ in order to keep the time interval $\Delta \tau$ between consecutive steps. 


\section{Non-linear vibrations of the bilinear structure: numerical simulations}

In the followings, the above described numerical simulation is applied. The dynamics of the structure due to the lift drag of vortex-shedding and a pedestrian flow is studied. The simulations are carried out with Maple 9.0 computer algebra system [18].

\subsection{Vertical vibrations due to vortex-shedding}

There are several effects that wind can exert on structures, such as vortex-shedding, flutter and buffeting [4]. Here the vertical force exerted on the beam by vortex-shedding [19, 20, 21, 22, 23, $24,25]$ is discussed.

It is assumed that the cable is neither stressed nor slacked in the initial (no-wind) state. The load has no static component, which implies $\alpha=0$ in Eq. (23), hence only a harmonic lift force occurs at the vortex-shedding frequency. This lift force can act either upward or downward, varying harmonically in time with the vortex-shedding frequency $[4,26]$ :

$$
f_{\mathrm{v}}=\frac{S t \cdot U}{D} \text {. }
$$

Here $S t$ is the Strouhal number (normally between $0.05-0.2$ ), $U$ is the wind flow velocity, and $D$ is the width of the frontal area of the deck. In the next section dimensionless numerical simulations are accomplished. The studied dimensionless time interval is $\tau \in[0,10]$ in the units of $T$, which is the forcing period. Then, the obtained results are validated with approximate hand calculations.

\subsubsection{Results}

In the following examples $\mathbf{a}=\mathbf{b}=\mathbf{0}$ in the initial time instant $\tau=0$. For a linear system it would imply harmonic, steady-state vibration with the forcing period. However, our structure is bilinear, and the lift force of the vortex-shedding may slack the suspension cable, which may lead to non-linear vibrations. Note that only the symmetric modal shapes play a role in the dynamic response of the structure. It is because of the zero initial values, the symmetric loading, and also because of symmetric modes are transformed into symmetric modes during state changes.

The unit of $\mu$ is chosen such that $q_{0} T^{2} /(\mu L)=1$. Hence only the frequency parameter $r$ can be varied. Six different values are studied, namely: $r=0.125,0.25,0.5,1,2$ and 4. A smaller value of $r$ corresponds to a softer beam. Table 1 shows the dimensionless natural periods of the symmetric active and passive modes for the studied values of $r$. Note that the dimensionless natural periods are in the units of $T$, and the dimensionless forcing period is one in the units of $T$. The frequency parameter is inversely proportional to the natural periods (see Eq. (22)). If a dimensionless natural period is greater than one, i.e. it is greater than the forcing period, then the forced vibration of the corresponding mode is out of phase. Note that in the case of $r=0.125,0.25$ and 0.5 the 1 st passive mode is out of phase.

Figure 4 shows the displacement of the mid point of the beam $u_{\text {mid }}$ versus dimensionless time $\tau$, while Figure 5 shows the velocity of the mid point of the beam versus its displacement for 


\begin{tabular}{ccccccccc}
\hline & & \multicolumn{7}{c}{ Dimensionless natural periods of symmetric active and passive modes } \\
$r$ & state & - & $T_{\mathrm{n}, 2}^{\mathrm{a}}$ & $T_{\mathrm{n}, 4}^{\mathrm{a}}$ & $T_{\mathrm{n}, 6}^{\mathrm{a}}$ & $T_{\mathrm{n}, 8}^{\mathrm{a}}$ & $T_{\mathrm{n}, 10}^{\mathrm{a}}$ & $T_{\mathrm{n}, 12}^{\mathrm{a}}$ \\
& & $T_{\mathrm{n}, 1}^{\mathrm{p}}$ & $T_{\mathrm{n}, 3}^{\mathrm{p}}$ & $T_{\mathrm{n}, 5}^{\mathrm{p}}$ & $T_{\mathrm{n}, 7}^{\mathrm{p}}$ & $T_{\mathrm{n}, 9}^{\mathrm{p}}$ & $T_{\mathrm{n}, 11}^{\mathrm{p}}$ & $T_{\mathrm{n}, 13}^{\mathrm{p}}$ \\
\hline \multirow{2}{*}{0.125} & active & - & 0.5658 & 0.3024 & 0.1241 & 0.06287 & 0.04997 & 0.03309 \\
& passive & 5.092 & 0.5658 & 0.2037 & 0.1039 & 0.06287 & 0.04209 & 0.03013 \\
\hline \multirow{2}{*}{0.25} & active & - & 0.2829 & 0.1512 & 0.06206 & 0.03143 & 0.02498 & 0.01654 \\
& passive & 2.546 & 0.2829 & 0.1018 & 0.05196 & 0.03143 & 0.02104 & 0.01506 \\
\hline \multirow{2}{*}{0.5} & active & - & 0.1414 & 0.07560 & 0.03103 & 0.01571 & 0.01249 & 0.008274 \\
& passive & 1.273 & 0.1414 & 0.05092 & 0.02598 & 0.01571 & 0.01052 & 0.007533 \\
\hline \multirow{2}{*}{1} & active & - & 0.07073 & 0.03780 & 0.01551 & 0.007859 & 0.006246 & 0.004137 \\
& passive & 0.6366 & 0.07073 & 0.02546 & 0.01299 & 0.007859 & 0.005261 & 0.003766 \\
\hline \multirow{2}{*}{2} & active & - & 0.03536 & 0.01890 & 0.007758 & 0.003929 & 0.003123 & 0.002068 \\
& passive & 0.3183 & 0.03536 & 0.01273 & 0.006496 & 0.003929 & 0.002630 & 0.001883 \\
\hline \multirow{2}{*}{4} & active & - & 0.01768 & 0.009450 & 0.003879 & 0.001964 & 0.001561 & 0.001034 \\
& passive & 0.1591 & 0.01768 & 0.006366 & 0.003248 & 0.001964 & 0.001315 & 0.0009417 \\
\hline
\end{tabular}

Table 1: Dimensionless natural periods of the relevant symmetric active and passive modes in the units of $T$ for the studied values of the frequency parameter $r$. Note that the 4th, 6th, 10th and 12th modes are the non-trivial active modes.

the studied values of the frequency parameter $r$. Since the initial values of $\mathbf{a}$ and $\mathbf{b}$ are zero, only forced vibration of the active modes occur in the beginning and free vibration is absent. Hence only in-phase, forced vibration of the active modes can be observed until $\tau=1 / 2$ in all the diagrams. Then the cable force vanishes and, due to the transition to passive modes, a combination of free and forced vibrations of symmetric passive modes evolves.

The smaller $r$ is the softer the structure is. For small $r$ the free vibration of the first (few) mode(s) governs the slow dynamics of the structure, while the higher modes and the forced response are the source of fast oscillations. However, if $r$ is larger (which means a stiffer structure), then the slow dynamics is governed by the forced response of the structure, and the fast oscillations are originated from the free vibrations of the modes.

Figures 4 (a) and 5 (a) correspond to $r=0.125$. The first passive dimensionless natural period, $T_{\mathrm{n}, 1}^{\mathrm{p}}=5.092$, can be well identified in the slow dynamics of the system with passive suspension: in the upward displacements in Figure 4 (a) and in the large ellipse in Figure 5 (a). The next period that is well observable is the unit period of the forcing, originating from the out-of-phase forcing of the passive modes. Natural vibrations of higher modes are also present as fast oscillations, but they are less apparent in the given scale. At approximately $\tau=1 / 2+T_{\mathrm{n}, 1}^{\mathrm{p}} / 2$ the suspension system becomes active again, and a combination of free and forced vibrations of the active modes occurs. The suspension remains active for about a $T_{\mathrm{n}, 2}^{\mathrm{a}} / 2$ time period, and it is inactivated again at about $\tau=1 / 2+T_{\mathrm{n}, 1}^{\mathrm{p}} / 2+T_{\mathrm{n}, 2}^{\mathrm{a}} / 2$. Then vibration occurs in the passive state for about a $T_{\mathrm{n}, 1}^{\mathrm{p}} / 2$ time period, then the suspension system is activated again, and so on. Since $r$ is small, and because 


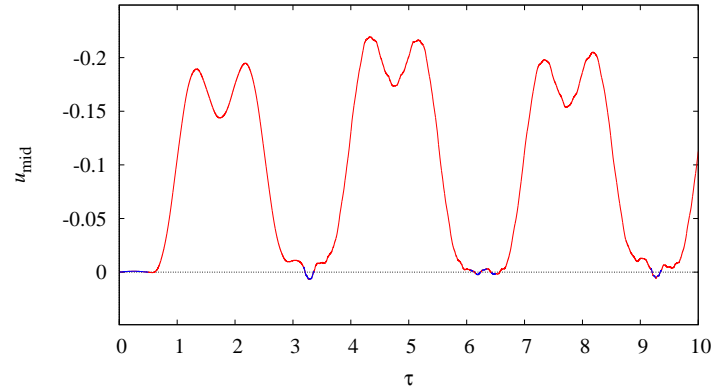

(a)

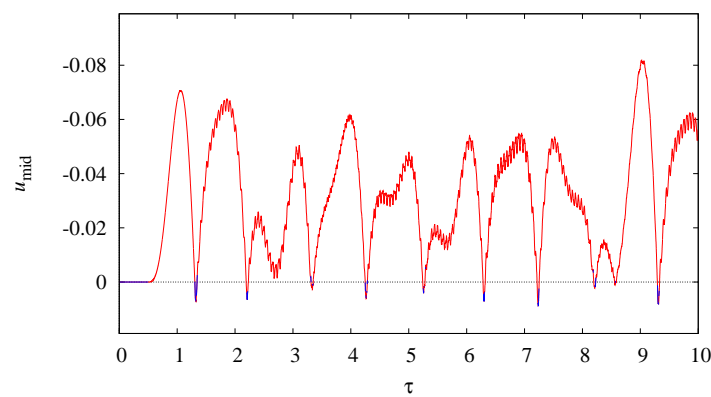

(c)

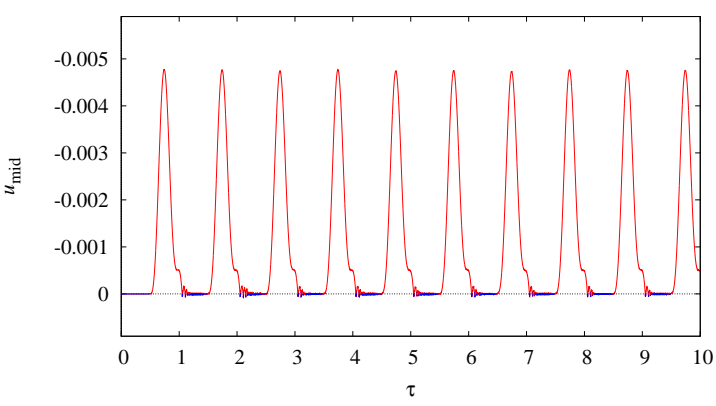

(e)

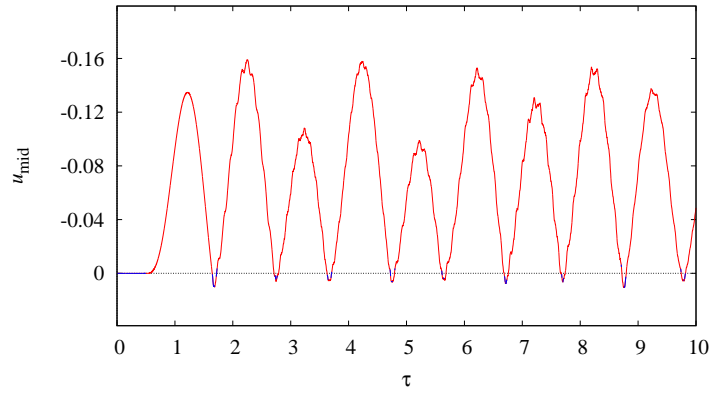

(b)

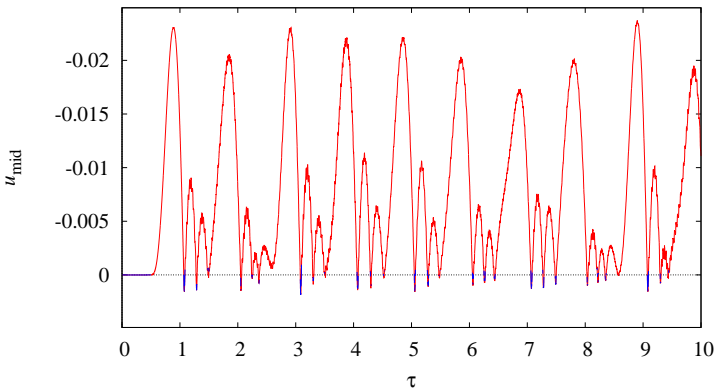

(d)

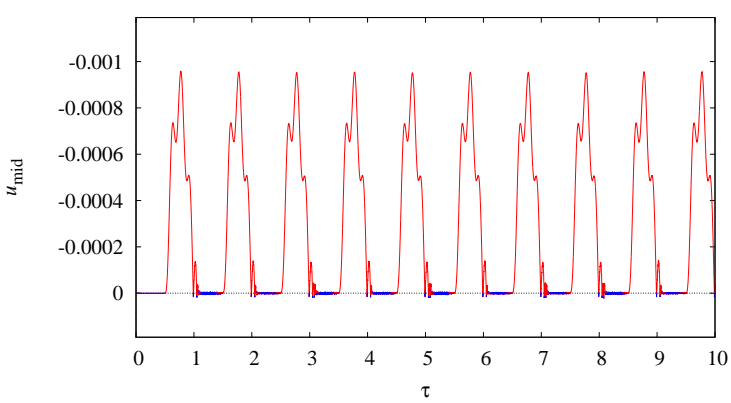

(f)

Figure 4: Vortex-shedding generated displacement of the mid point of the beam $u_{\text {mid }}$ as the function of dimensionless time $\tau$ for frequency parameters $r=$ (a) 0.125 , (b) 0.25 , (c) 0.5 , (d) 1 , (e) 2, and (f) 4 . Blue and red colors belong to active and passive suspension, respectively. $q_{0} T^{2} /(\mu L)=1, \Delta \tau=0.0005$. 


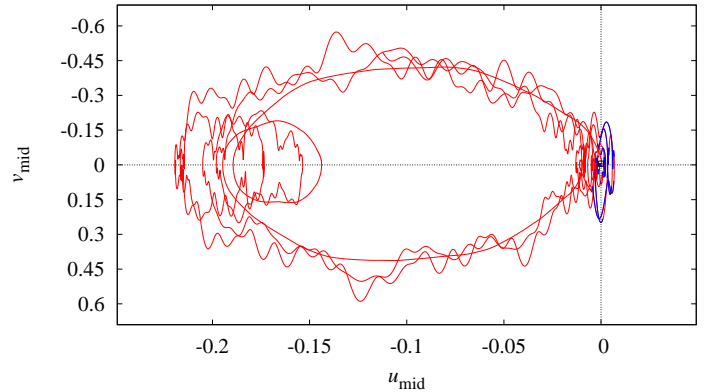

(a)

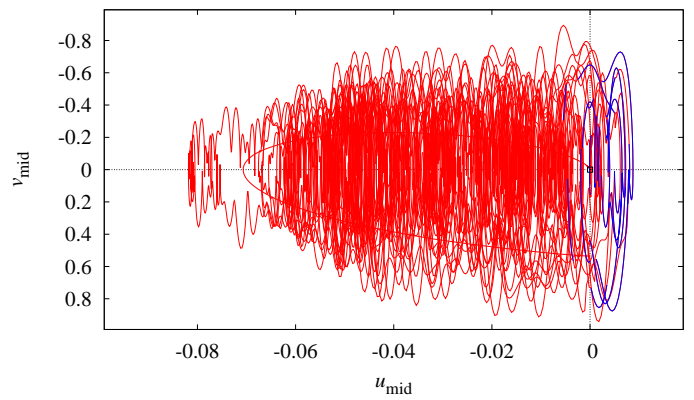

(c)

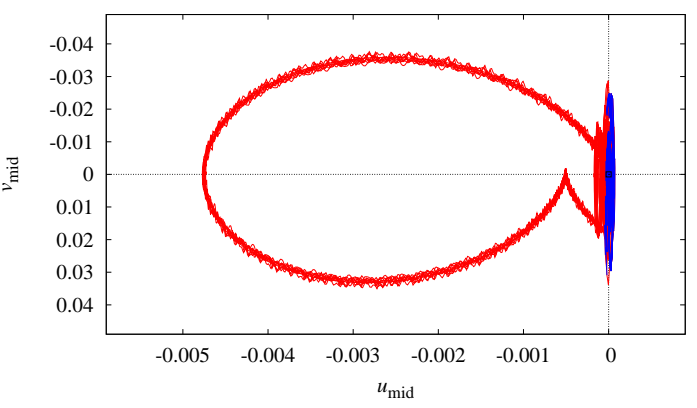

(e)

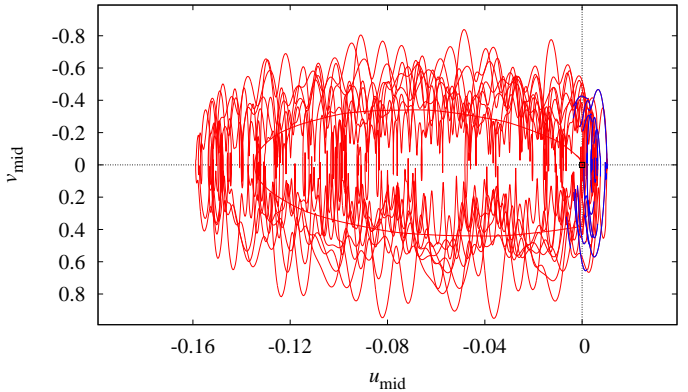

(b)

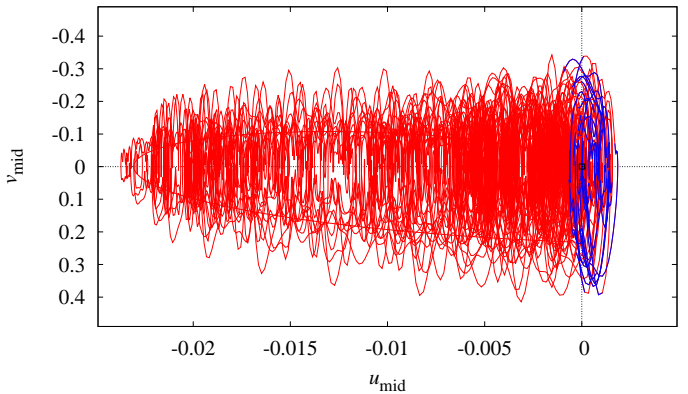

(d)

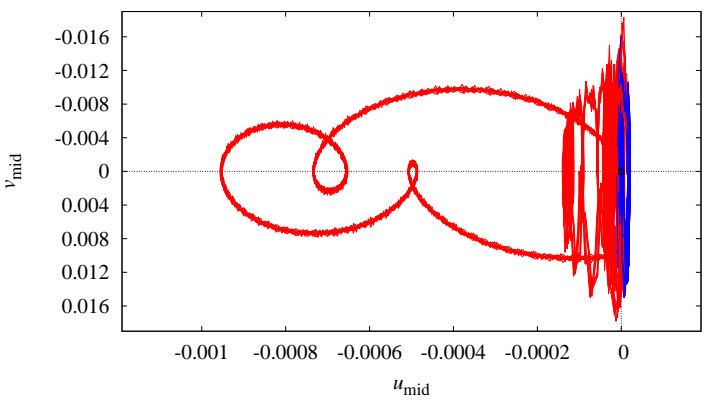

(f)

Figure 5: Vortex-shedding generated velocity-displacement diagrams of the mid point of the beam for frequency parameters $r=$ (a) 0.125 , (b) 0.25 , (c) 0.5 , (d) 1 , (e) 2, and (f) 4 . Blue and red colors belong to active and passive suspension, respectively. $q_{0} T^{2} /(\mu L)=1, \Delta \tau=0.0005$. 
the structure is approximately 10 times stiffer in the active state than in the passive state, it spends around 10 times less time in the active state than in the passive state. Hence, the period of this forced vibration, for $r=0.125$, is roughly:

$$
T_{\mathrm{f}}=\frac{11}{20} T_{\mathrm{n}, 1}^{\mathrm{p}} .
$$

Figures 4 (b) and 5 (b) correspond to $r=0.25$, where $T_{\mathrm{n}, 1}^{\mathrm{p}}=2.546$. This natural period can be clearly seen in the large ellipse in Figure 5 (b). The upward displacement is not a pure half sine wave with period $T_{\mathrm{n}, 1}^{\mathrm{p}} / 2$, but distorted slightly by the forced response of the passive modes. The free vibrations of higher passive modes are the source of fast oscillations. The time period that the structure spends in the passive state varies around 1. The vibration of the structure in the active state is mostly dominated by the 1 st symmetric active mode, which has the natural period of $T_{\mathrm{n}, 2}^{\mathrm{a}}=0.2829$.

Figures 4 (c) and 5 (c) correspond to $r=0.5$. The 1 st passive natural period, $T_{\mathrm{n}, 1}^{\mathrm{p}}=1.273$, is not far from the unit forcing period. Here the free and the out-of-phase forced vibrations of the 1st passive mode compete in the large upward displacements, while the free vibrations of higher modes can be observed as short "ripples" (fast oscillations). An animation of the vibrating beam can be found online, see Appendix A.

Figures 4 (d) and $5(\mathrm{~d})$ belong to $r=1$. Here $T_{\mathrm{n}, 1}^{\mathrm{p}}=0.6366$, which is smaller than the forcing period, thus all the forced vibration modes are in phase. The period of the large tongues upward is approximately one, and these forced responses of the passive system compete with the free vibration modes. An animation of the vibrating beam is available online, see Appendix A.

In the previous two examples it is not possible to give a good approximation for the period of the vibration, since the vibration does not seem to be periodic, it is rather quasi-periodic or chaotic.

The next two diagrams correspond to fairly large values of $r$, i.e. to stiffer structures. Figures 4 (e) and 5 (e) correspond to $r=2$. Here the forced vibration of unit period dominates the response of the structure both in the active and in the passive states. The forced response of the structure is apparent in the slow dynamics, while the free vibration modes are present as fast oscillations. The same pattern can be observed for the case of $r=4$. This is shown in Figures 4 (f) and 5 (f). The structure is even more rigid. The time interval between state changes apparently tends to $1 / 2$, indicating that the slow dynamics is governed by the forced response of the structure. Hence, in these two examples the period of vibration is approximately:

$$
T_{\mathrm{f}}=1 \text {. }
$$

Finally, it is concluded that the dynamics of a soft suspended beam subjected to harmonic vertical excitation is governed by the natural vibration of its first passive mode. The structure spends much less time in the active state than in the passive state, and its period of vibration tends to (36) as $r$ decreases. However, if the suspended beam is stiffer, then its dynamics is dominated by the forced vibration, the system spends about the same amount of time in the active state as 
in the passive state, and the period of vibration tends to (37) as $r$ increases. If the structure is in between these two extremities, then it can undergo complicated non-linear vibrations.

\subsubsection{Verification}

In this section the numerical simulation is validated by applying basic formulas and concepts from the literature $[14,27,26]$ to a realistic structure and comparing the results with the numerical outcomes of the previous section. A simple foot bridge with total length $L=18 \mathrm{~m}$, width $w=4 \mathrm{~m}$, and height of frontal are $D=0.75 \mathrm{~m}$ is studied. There are two main girders, each equipped by a block-and-tackle suspension system. Because the flexural-torsional and the lateral vibrations are restricted, only one half of the bridge is analysed: one girder, the half of the deck and secondary structures, and one suspension system. The girder is an IPE270 steel beam with bending stiffness $E I=12 \mathrm{MNm}^{2}$. The mass per unit length, including half of all the load bearing and secondary structures, is taken to be $\mu=455 \mathrm{~kg} / \mathrm{m}$. The Strouhal number, $S t=0.1$, and the lift coefficient, $C_{L}=\sqrt{2} \cdot 0.6=0.85$, are set according to [26] p. 29.35-29.37. The studied wind velocity is $U=7.5 \mathrm{~m} / \mathrm{s}$, the density of air is $\rho=1.29 \mathrm{~kg} / \mathrm{m}^{3}$. The vortex-shedding frequency is $f=1 \mathrm{~Hz}$, according to (35), and so the reference time period of our model is:

$$
T=1 / f=1 \mathrm{~s} .
$$

The harmonic lift load per unit length is given by [26]:

$$
q_{\mathrm{v}}(t)=\frac{1}{2} \rho U^{2} C_{L} D \sin (2 \pi f t)=q_{\mathrm{v} 0} \cdot \sin (2 \pi t), \quad \text { where } \quad q_{\mathrm{v} 0}=23.13 \mathrm{~N} / \mathrm{m} .
$$

The vibration of the structure without suspension system due to the upward lift load (38) is approximated by a single-degree-of-freedom model. The bridge with slacked cable is a simply supported beam, which has the fundamental natural period [14]:

$$
\hat{T}_{\mathrm{n}}=2 \pi \frac{L^{2}}{\pi^{2}} \sqrt{\frac{\mu}{E I}}=1.270 \mathrm{~s},
$$

and the static mid-point deflection ([27], p. 536):

$$
\hat{u}_{\mathrm{st}}^{\mathrm{app}}=\frac{5 \cdot q_{\mathrm{v} 0} \cdot L^{4}}{384 \cdot E I}=2.635 \cdot 10^{-3} \mathrm{~m} .
$$

The deformation response factor (or magnification factor [14]) yields:

$$
R=\frac{1}{1-\left(\hat{T}_{\mathrm{n}} / T\right)^{2}}=-1.6316
$$

Finally, the approximate dynamic deflection of the mid point of the beam, considering only the 
particular solution and the fundamental mode of the forced vibration is:

$$
\hat{u}_{\mathrm{dyn}}^{\mathrm{app}}=|R| \cdot \hat{u}_{\mathrm{st}}^{\mathrm{app}}=4.299 \cdot 10^{-3} \mathrm{~m} .
$$

Now the dynamic deflection of the model is computed, using the concepts developed in the paper for passive suspension system. The frequency parameter of the structure is from (6):

$$
r=\frac{T}{L^{2}} \sqrt{\frac{E I}{\mu}}=0.5012 .
$$

Hence, the results in Sec. 5.1.1 for $r=0.5$ can be directly applied. The first natural period of passive state for this frequency parameter is given in Table 1 in the units of the reference time period $T$. Its real value is:

$$
\hat{T}_{\mathrm{n}, 1}^{\mathrm{p}}=T_{\mathrm{n}, 1}^{\mathrm{p}} \cdot T=1.273 \mathrm{~s} .
$$

Note that the difference between (39) and (42) is caused by the small deviation of (41) from $r=$ 0.5. Figure 4 (c) shows the dimensionless displacement of the mid point of the beam versus the dimensionless time. The maximum upward displacement that corresponds to the passive state of the structure is:

$$
u_{\mathrm{dyn}}^{q_{0}}=0.08185 .
$$

Since $q_{0} T^{2} /(\mu L)=1$ was set in the simulations, the distributed load amplitude that the above result corresponds to is:

$$
q_{0}=\frac{\mu L}{T^{2}}=8190 \mathrm{~N} / \mathrm{m} \text {. }
$$

Finally, the obtained displacement (43) is scaled to the lift force amplitude (38) and its real value is computed:

$$
\hat{u}_{\mathrm{dyn}}=u_{\mathrm{dyn}}^{q_{0}} \frac{q_{\mathrm{v} 0}}{q_{0}} L=4.161 \cdot 10^{-3} \mathrm{~m} .
$$

There is about 3\% difference between Eqs. (40) and (44). The following reasons lie behind it. First, the approximate analysis incorporated the fundamental vibration mode, while our model also took into account higher modes, which are in-phase, contrary to the first mode, which is outof-phase. Second, our simulation inherits a slight numerical damping at each transition. Third, our simulation takes into account the free vibration of the structure, too. In view of these, the two results are in a surprisingly good agreement. Of course, there can be huge differences in other quantities, like the internal forces.

Finally, let us fix the period of time $T$, and mass $\mu$ and length $L$ of the beam, and give the bending stiffness $E I$ corresponding to the frequency parameters studied in the previous section. The bending stiffness values are $0.75,3,12,48,192$ and $764 \mathrm{MNm}^{2}$ corresponding to the frequency parameter values $0.125,0.25,0.5,1,2$, and 4 , respectively. 


\subsection{Pedestrian flow induced vertical vibrations}

The passenger traffic load has both static and dynamic components. According to technical guidelines $[28,29]$, the step frequency of normal walking is approximately $f=2 \mathrm{~Hz}$, although there are also higher harmonics [30]. Here a continuous pedestrian stream is considered: the passenger traffic load is modelled by a static plus a harmonic distributed load. Guidelines [28, 29] suggest that the amplitude of the dynamic load is $10 \%$ of the static load. It implies that $\alpha=10$ in Eq. (23). It is assumed that the cable is neither slacked nor stressed when the beam is free from pedestrians. The static component of the pedestrian flow applies a static tension in the suspension cable, while the dynamic component may make the cable more stressed, less stressed, or slacked. If the effect of the dynamic load is small, then it cannot slack the cable and the suspension system remains active during vibration. However, if the dynamic effect is large enough, then non-linear vibrations can occur due to consecutive cable slacking and stressing. Such a resonant behavior typically happens when the walking frequency is close to one of the natural frequencies of the structure. In the next section dimensionless numerical simulations are accomplished. The studied dimensionless time interval is $\tau \in[0,50]$ in the units of $T$, which is the forcing period. Then, the obtained results are validated.

\subsubsection{Results}

The unit of $\mu$ can be chosen such that $q_{\mathrm{st}} T^{2} /(\mu L)=1$. The ratio of the static component to the dynamic amplitude is already set, $\alpha=10$. Therefore, only the frequency parameter $r$ can be varied. Because of the loading is symmetric, and the initial values are zero, only the symmetric modal shapes appear in the structural response. Note that the forcing period is always one in the units of $T$, according to (19).

The first non-trivial active mode is the 4 th mode, which is symmetric. The natural frequency of this mode coincides with the forcing frequency if $f_{4}^{a}=1 / T$ in (8). The corresponding frequency parameter is:

$$
r_{\text {res }}=0.03780 \text {. }
$$

Then the dimensionless natural periods of vibration in the units of $T$ are $T_{\mathrm{n}, 1}^{\mathrm{p}}=16.84$ for the $1 \mathrm{st}$ passive mode, $T_{\mathrm{n}, 2}^{\mathrm{a}}=T_{\mathrm{n}, 3}^{\mathrm{p}}=1.871$ for the 2 nd active and 3 rd passive modes, and $T_{\mathrm{n}, 4}^{\mathrm{a}}=1$ for the (resonant) 4th active mode.

In the following examples the frequency parameter $r$ is tuned close to this resonant state and numerical simulations are accomplished with initial values $\mathbf{a}=\mathbf{b}=\mathbf{0}$. The deformation response factor (25) of the 4th active mode is prescribed as $R_{\mathrm{d}, 4}^{\mathrm{a}}=10,15,20,-20,-15$ and -10 . The corresponding frequency parameters are computed from:

$$
r=r_{\text {res }} \sqrt{\frac{R_{\mathrm{d}, 4}^{\mathrm{a}}}{R_{\mathrm{d}, 4}^{\mathrm{a}}-1}} .
$$

Table 2 shows the studied frequency parameter values and the deformation response factors of the symmetric active and passive modes. 


\begin{tabular}{ccccccccc}
\hline & & \multicolumn{6}{c}{ Deformation response factors of symmetric active and passive modes } \\
$r$ & state & - & $R_{\mathrm{d}, 2}^{\mathrm{a}}$ & $R_{\mathrm{d}, 4}^{\mathrm{a}}$ & $R_{\mathrm{d}, 6}^{\mathrm{a}}$ & $R_{\mathrm{d}, 8}^{\mathrm{a}}$ & $R_{\mathrm{d}, 10}^{\mathrm{a}}$ & $R_{\mathrm{d}, 12}^{\mathrm{a}}$ \\
& & $R_{\mathrm{d}, 1}^{\mathrm{p}}$ & $R_{\mathrm{d}, 3}^{\mathrm{p}}$ & $R_{\mathrm{d}, 5}^{\mathrm{p}}$ & $R_{\mathrm{d}, 7}^{\mathrm{p}}$ & $R_{\mathrm{d}, 9}$ & $R_{\mathrm{d}, 11}^{\mathrm{p}}$ & $R_{\mathrm{d}, 13}^{\mathrm{p}}$ \\
\hline \multirow{2}{*}{0.03985} & active & - & -0.4648 & $\mathbf{1 0}$ & 1.178 & 1.040 & 1.025 & 1.011 \\
& passive & -0.003932 & -0.4648 & 1.690 & 1.119 & 1.040 & 1.017 & 1.009 \\
\hline \multirow{2}{*}{0.03912} & active & - & -0.4408 & $\mathbf{1 5}$ & 1.186 & 1.042 & 1.026 & 1.011 \\
& passive & -0.003792 & -0.4409 & 1.735 & 1.124 & 1.042 & 1.018 & 1.009 \\
\hline \multirow{2}{*}{0.03878} & active & - & -0.4298 & $\mathbf{2 0}$ & 1.190 & 1.043 & 1.026 & 1.011 \\
& passive & -0.003725 & -0.4298 & 1.758 & 1.126 & 1.043 & 1.019 & 1.009 \\
\hline \multirow{2}{*}{0.03689} & active & - & -0.3736 & $\mathbf{- 2 0}$ & 1.215 & 1.047 & 1.029 & 1.013 \\
& passive & -0.003369 & -0.3736 & 1.910 & 1.141 & 1.047 & 1.021 & 1.010 \\
\hline \multirow{2}{*}{0.03660} & active & - & -0.3656 & $\mathbf{- 1 5}$ & 1.219 & 1.048 & 1.030 & 1.013 \\
& passive & -0.003316 & -0.3656 & 1.938 & 1.144 & 1.048 & 1.021 & 1.010 \\
\hline \multirow{2}{*}{0.03604} & active & - & -0.3506 & $\mathbf{- 1 0}$ & 1.227 & 1.050 & 1.031 & 1.013 \\
& passive & -0.003215 & -0.3506 & 1.997 & 1.149 & 1.050 & 1.021 & 1.011 \\
\hline
\end{tabular}

Table 2: Deformation response factors of symmetric active and passive modes for the studied values of the frequency parameter $r$. Note that the non-trivial active modes are the 4th, 6th, 10th and 12th ones.

For the studied values of the frequency parameter $r$, Figure 6 shows the displacement of the mid point of the beam $u_{\text {mid }}$ versus the dimensionless time $\tau$, while Figure 7 shows the velocity of the mid point of the beam $v_{\text {mid }}$ versus its displacement $u_{\text {mid }}$. The dynamics starts with the initial static deflection and the velocity corresponds to zero initial values of $\mathbf{a}$ and $\mathbf{b}$. Forced vibration of the active modes occurs, free vibration is absent. If the system is close enough to the state of resonance, then the cable force vanishes after a while and the suspension system becomes passive. Some time later the suspension system will be active again, but it can be inactivated later, and so on. These consecutive transitions between active and passive states may lead to non-linear vibrations.

Figures 6 (a) and 7 (a) correspond to $R_{\mathrm{d}, 4}^{\mathrm{a}}=10(r=0.03985)$. A steady-state harmonic vibration occurs in this case with the unit (dimensionless) period of the forcing. The dynamic effect of the pedestrian flow is not enough to slack the cables and hence do not cause non-linear vibrations.

Figures 6 (b) and 7 (b) correspond to a slightly more flexible structure: $R_{\mathrm{d}, 4}^{\mathrm{a}}=15(r=$ 0.03912). At the beginning of the time-displacement diagram (for $\tau<10$ ), one may assume a harmonic vibration with the unit time period of the forcing. The colors, however, reveal that trabsitions between active and passive states occur, and also the amplitude of the displacement slightly increases with time. Later the variation in the amplitude is more apparent. The displacementvelocity diagram is not an ellipse, as in the previous example, but some deviation is observed. Hence, this forced vibration has a non-linear nature.

Figures 6 (c) and 7 (c) correspond to $R_{\mathrm{d}, 4}^{\mathrm{a}}=20(r=0.03878)$, i.e. to a structure slightly more flexible than the previous one. The period of vibration closely follows the unit forcing period. In 


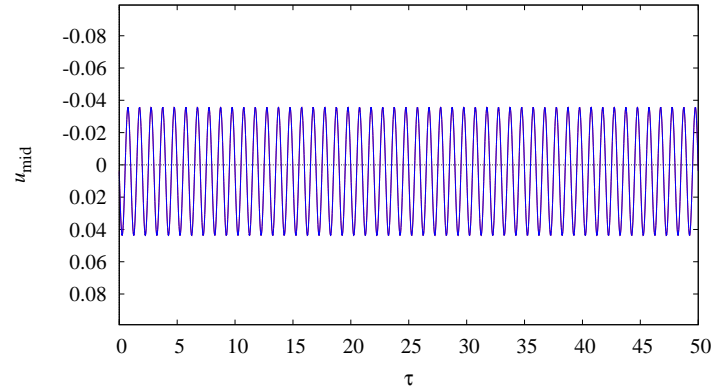

(a)

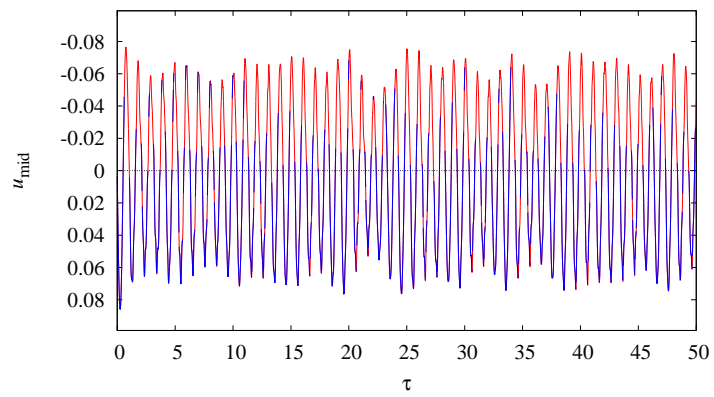

(c)

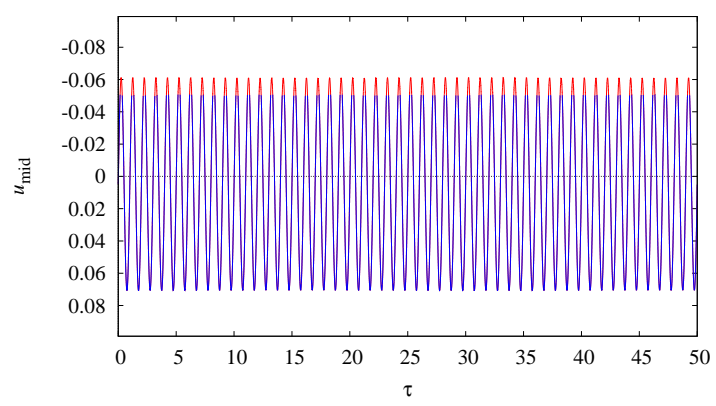

(e)

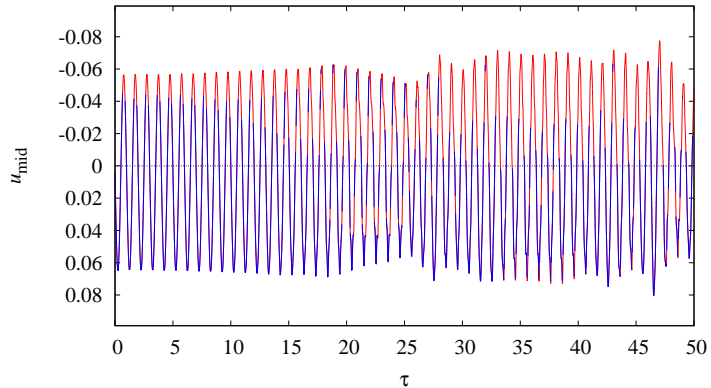

(b)

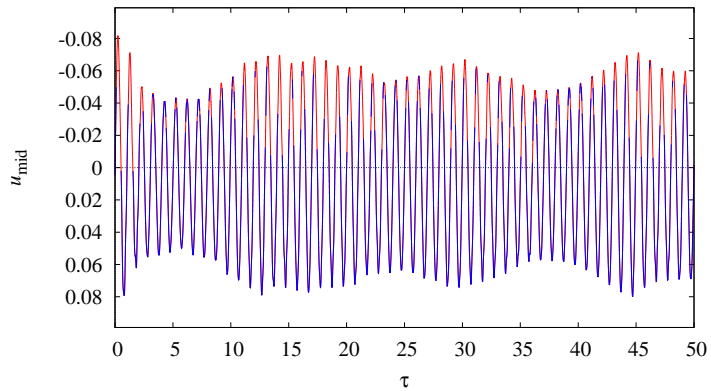

(d)

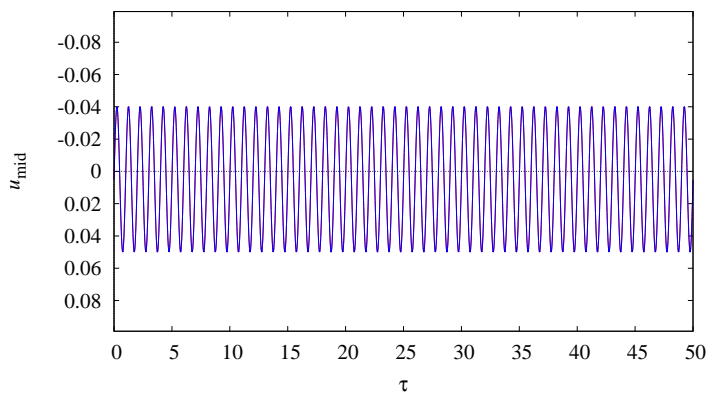

(f)

Figure 6: Pedestrian flow induced displacement of the mid point of the beam $u_{\text {mid }}$ as the function of dimensionless time $\tau$ for frequency parameters $r=$ (a) 0.03985, (b) 0.03912, (c) 0.03878, (d) 0.03689, (e) 0.03660, and (f) 0.03604 . Blue and red colors belong to active and passive suspension, respectively. $q_{\mathrm{s}} T^{2} /(\mu L)=1, \alpha=10, \Delta \tau=0.001$. 


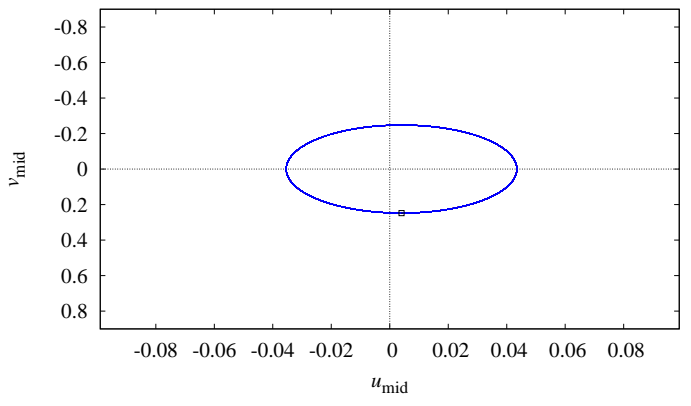

(a)

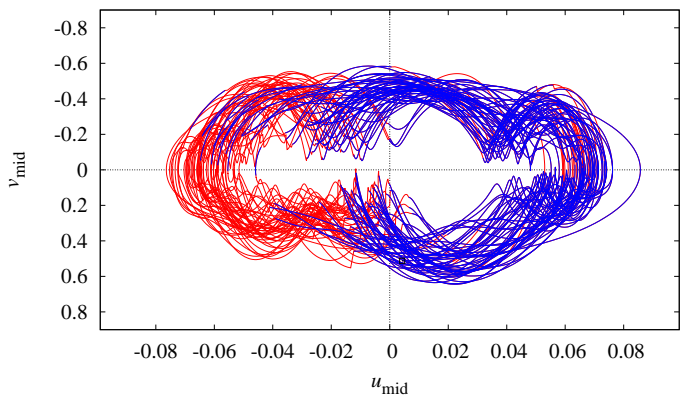

(c)

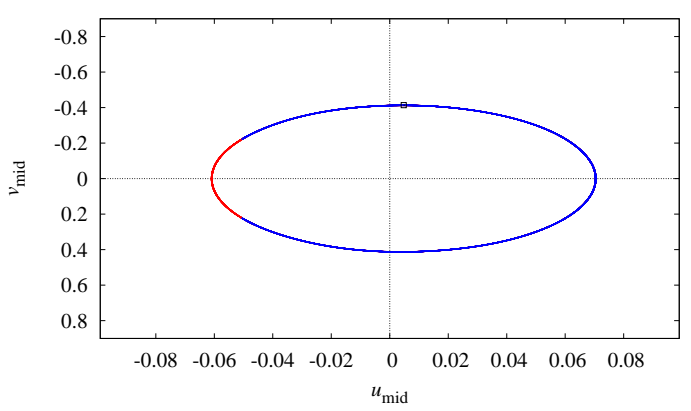

(e)

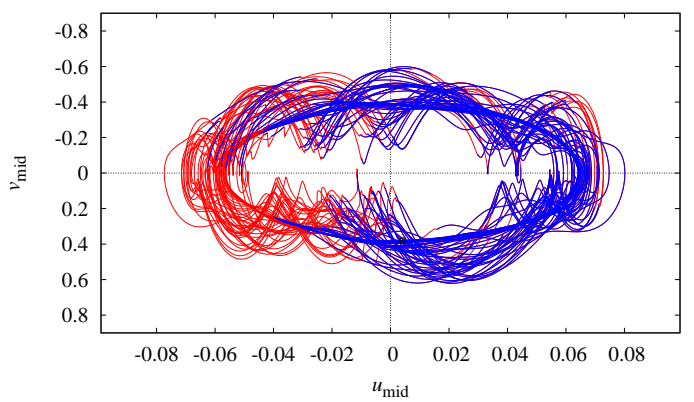

(b)

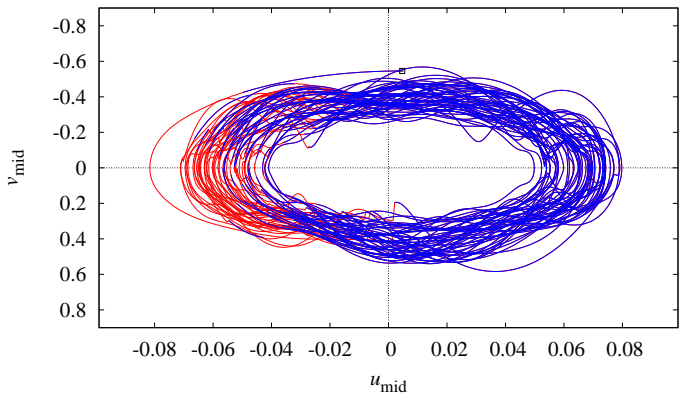

(d)

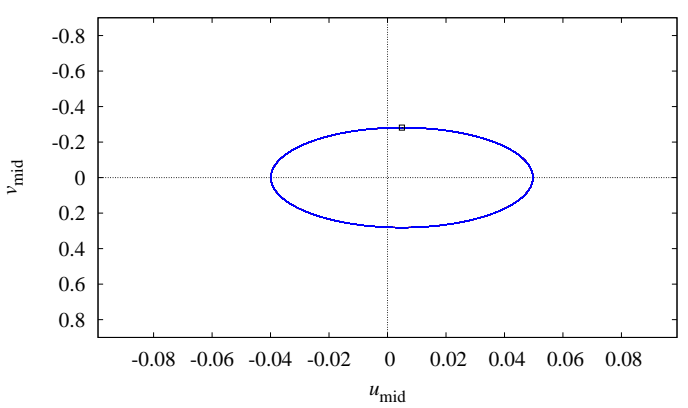

(f)

Figure 7: Pedestrian flow induced velocity-displacement diagrams of the mid point of the beam for frequency parameters $r=$ (a) 0.03985, (b) 0.03912, (c) 0.03878, (d) 0.03689, (e) 0.03660, and (f) 0.03604. A box denotes the initial state $(\tau=0)$. Blue and red colors belong to active and passive suspension, respectively. $q_{\mathrm{s}} T^{2} /(\mu L)=1, \alpha=$ $10, \Delta \tau=0.001$. 
the time-displacement diagram the time intervals between the consecutive zeros are $\approx 1 / 2$, but the curve is not a pure sine wave, the amplitude varies, the effects of transitions and the free vibration modes can be observed. In the displacement-velocity diagram an averaged ellipse could be drawn around which faster oscillations occur. Note how mixed the active and passive states are in this diagram.

Figures $6(\mathrm{~d})$ and $7(\mathrm{~d})$ correspond to $R_{\mathrm{d}, 4}^{\mathrm{a}}=-20(r=0.03689)$. The deviation of the dynamics from a simple harmonic motion is still apparent, but the system spends a shorter time in the passive state and transitions occur less frequently than in the previous case. A possible reason of it is that the 4th active mode is out of phase now, while the higher modes are in phase, and they can partially compensate the effect of the 4th resonant mode. (Although the 2 nd active mode is out of phase, it has a much smaller deformation response factor than the higher modes.) In the previous example, however, the dynamic effect of the resonant in-phase 4th mode is supported by the in-phase higher modes.

Figures 6 (e) and 7 (e) belong to an even more flexible structure, where $R_{\mathrm{d}, 4}^{\mathrm{a}}=-15(r=$ 0.03660). Here it can be observed that the structure undergoes a periodic motion with unit time period, although the suspension system is activated regularly for about $1 / 10$ long time within each period. This is an exceptional case: the structure is bilinear, but the cable stressing and slacking, and the structural vibration are periodic. A possible reason behind this phenomenon can be that the higher, in-phase active modes balance the 4th resonant, out-of-phase mode.

Figures 6 (f) and 7 (f) correspond to the most flexible studied structure, where $R_{\mathrm{d}, 4}^{\mathrm{a}}=-10$ $(r=0.03604)$. Here the out-of-phase forced vibration of the 4th active mode is not large enough to slack the cables. Hence harmonic vibrations of the active states occur with a unit (dimensionless) time period, which appears to be a sine wave in the time-displacement diagram and an ellipse in the displacement-velocity diagram.

Whether the cable force vanishes or not determines if the structure may exhibit non-linear vibrations or not. The question is if the tensile cable force from the static load is enough to keep the suspension system active during the vibration. Cable force arises from non-trivial active modes only. An approximate condition for cable slacking estimates the cable force from the static and dynamic loads and compares them. This condition can be written as:

$$
\alpha\left|\sum_{\text {non-triv. }} h_{i}^{\mathrm{a}}\right|<\left|\sum_{\text {non-triv. }} R_{\mathrm{d}, i}^{\mathrm{a}} h_{i}^{\mathrm{a}}\right| .
$$

Here $h_{i}^{\mathrm{a}}$ and $R_{\mathrm{d}, i}^{\mathrm{a}}$ are given by (26) and (25), respectively. If the above inequality holds, then transitions between active and passive suspension states can be expected, which are the source of the non-linear dynamics.

\subsubsection{Verification}

In this section the numerical simulation is verified. A simple foot bridge is analysed with total length $L=18 \mathrm{~m}$ and width $w=4 \mathrm{~m}$, composed of two main (fairly flexible) girders and two 
suspension systems. The static pedestrian surface load [28] is: $375 \mathrm{~N} / \mathrm{m}^{2}$. Ten percent of this load is the dynamic load amplitude. One half of the bridge is studied: one girder, the half of the deck and secondary structures, and one suspension system. The bending stiffness of the girder is taken to be $E I=0.3 \mathrm{MNm}^{2}$, the mass per unit length is $\mu=450 \mathrm{~kg} / \mathrm{m}$. The static line load is the surface load times $w / 2$ :

$$
q_{\mathrm{p}}=750 \mathrm{~N} / \mathrm{m} \text {. }
$$

The time period of the dynamic load component corresponds to the walking frequency $f=$ $2 \mathrm{~Hz}$, hence

$$
T=1 / f=0.5 \mathrm{~s} .
$$

The frequency parameter of the structure is computed according to Eq. (6):

$$
r=\frac{T}{L^{2}} \sqrt{\frac{E I}{\mu}}=0.03985 .
$$

Based on the corresponding result of the simulation, shown in Figure 6 (a), the dimensionless initial static displacement is $u_{\text {stat }}=0.004027$, while the maximal dynamic displacement is $u_{\text {dyn }}=$ 0.04346. Since $q_{\mathrm{s}} T^{2} /(\mu L)=1$, the dimensionless results correspond to the load $q_{\mathrm{s}}=32400$. Therefore, the real values of the static (initial) and dynamic (maximal) downward displacement of the mid point of the beam are:

$$
\begin{aligned}
& \hat{u}_{\text {stat }}=u_{\text {stat }}^{\text {mid }} \frac{q_{\mathrm{p}}}{q_{\mathrm{s}}} L=1.678 \cdot 10^{-3} \mathrm{~m}, \\
& \hat{u}_{\text {dyn }}=u_{\text {dyn }}^{\text {mid }} \frac{q_{\mathrm{p}}}{q_{\mathrm{s}}} L=1.811 \cdot 10^{-2} \mathrm{~m} .
\end{aligned}
$$

Note that Eq. (18) gives the same static displacement of the same structure for the same distributed load level, and so it verifies Eq. (45).

It is a bit more difficult to verify the maximal dynamic displacement. since the resonant fourth mode has the most important dynamic effect, the other modes are neglected. The fourth active modal shape coincides with the third mode shape of a continuous three-span-beam. The natural frequencies of such a beam are tabulated in [26] at p. 7.24, and the corresponding period verifies our result based on (22):

$$
\hat{T}_{\mathrm{n}, 4}^{\mathrm{a}}=T_{\mathrm{n}, 4}^{\mathrm{a}} \cdot T=0.9487 \mathrm{~s} .
$$

The deformation response factor for this mode is:

$$
R_{\mathrm{d}, 4}^{\mathrm{a}}=\frac{1}{1-\left(\hat{T}_{\mathrm{n}, 4}^{\mathrm{a}} / T\right)^{2}}=9.999
$$

The normalized fourth shape function of the active state evaluated at the mid point of the beam is: $u_{4}^{\text {mid }}=2.098$. (See Figure 2.) Using Eq. (17), $\eta_{4}^{\mathrm{s}}=4.176 \cdot 10^{-5}$ with the dynamic load amplitude 
$q_{0}=75 \mathrm{~N} / \mathrm{m}$. (The integral of the fourth modal shape function is $F_{4}^{\mathrm{a}}=0.7916$.) Thus, the dimensionless static displacement due to the dynamic part of the pedestrian load, corresponding to the resonant mode is: $u_{\text {stat }, 4}=\eta_{4}^{\mathrm{s}} \cdot u_{4}^{\mathrm{mid}}=8.761 \cdot 10^{-5}$. If multiplied by $L$, its real value is obtained:

$$
\hat{u}_{\text {stat }, 4}=0.001577 \mathrm{~m} \text {. }
$$

Finally, the approximate maximal displacement of the mid point of the beam, due to the static and dynamic parts of the pedestrian flow is:

$$
\hat{u}_{\mathrm{dyn}}^{\mathrm{app}}=\hat{u}_{\mathrm{stat}}+R_{\mathrm{d}, 4}^{\mathrm{a}} \cdot \hat{u}_{\mathrm{stat}, 4}=1.745 \cdot 10^{-2} \mathrm{~m} .
$$

The terms before the last equal sign are the followings. The first term is the static displacement due to the static part of the pedestrian flow. The second term is the displacement caused by the dynamic part of the pedestrian flow, taking into account only the resonant mode. Eq. (47) is a good approximate for Eq. (46), regarding that it neglects the dynamic deformations from the nonresonant modes. Thus, it validates the numerical algorithm.

\section{Conclusions}

In this paper the dynamics of a simply supported Bernoulli-Euler beam equipped with a blockand-tackle suspension system was studied. It is an effective, innovative suspension (foot)bridge model, which allows a uniform cable force distribution. As the suspension cable cannot resist compression, the suspension system can be either in active or in passive state. Because of this, the studied structure can be described by a piece-wise linear system, which can be a source of nonlinear vibrations. The frequency parameter of the structure was introduced, and the dimensionless natural frequencies and modal shape functions were derived for both stressed and slacked cable. A procedure was developed to handle the nonlinearity originating from the bilinear stiffness characteristic of the structure. A numerical damping phenomenon was revealed during the transition between active and passive states, which was caused by the finitization of the space of the modal shapes. Then semi-analytical simulations were accomplished. Three types of loadings were studied: a static distributed load for verification purposes, a harmonic distributed load for modelling a vortex-shedding generated lift force, and a static plus harmonic distributed load for modelling the dynamic effects of a pedestrian flow. In the case of the vortex-shedding load non-linear vibrations were observed, which were induced by consecutive slacking and stressing of the suspension cable. It was pointed out that the frequency parameter is the only parameter that determines the structural vibration qualitatively. It was revealed that for a soft structure the slow dynamics was governed by the free vibration, and fast oscillations evolved from the forced vibration. On the other hand, for a stiff structure, slow dynamics originated from the forced vibration while the fast oscillations were due to the free vibrations of the natural modes. Estimates for the period of vibration of these two extremities were given. In the case of the pedestrian flow, by fixing the ratio of the static and dynamic load components as given by guidelines, only the frequency parameter was needed for the 
dimensionless analysis of the vibrations. It was shown that the natural frequency of one non-trivial active mode had to be close enough to the step frequency of walking in order to slack the cable, otherwise harmonic motion occurred. Six different frequency parameter values were studied. Two of them led to linear vibrations, but in the other four cases non-linear vibrations occurred. The outcomes of the numerical simulations were thoroughly discussed and a necessary condition for cable slacking was developed. The numerical results were validated by hand calculations, and it was demonstrated how to apply the dimensionless forms to real structures.

In future works it would be worth studying the effects of structural damping, the mass of the cable, the friction at the pulleys, or different arrangements of the pulleys. By taking into account fewer natural modes, a global non-linear dynamic analysis can also be an interesting continuation of the present work.

\section{Acknowledgement}

The work of A. Kocsis was supported by the János Bolyai Research Scholarship of the Hungarian Academy of Sciences and by OTKA No. PD100786. Financial support from OTKA No. K100894 is also gratefully acknowledged. We thank György Károlyi for valuable discussions.

\section{Appendix A. Supplementary material}

Supplementary material associated with this article can be found in the online version.

\section{References}

[1] J. O'Kon, Computer modeling of the seventh century Maya suspension bridge at Yaxchilan, Proceedings of the 2005 ASCE International Conference on Computing in Civil Engineering 179 (124) (2005) 1-14.

[2] F. Veranzio, Machinae Novae, Venice, 1595.

[3] T. Kawada, History of the Modern Suspension Bridge, ASCE Press, Reston, Virginia, 2010.

[4] J. D. Holmes, Wind Loading of Structures, Taylor \& Francis, London, 2007.

[5] Y. Tamura, A. Kareem, Advanced Structural Wind Engineering, Springer, Japan, 2013.

[6] E. T. Ingólfsson, C. T. Georgakis, J. Jönsson, Pedestrian-induced lateral vibrations of footbridges: A literature review, Engineering Structures 45 (2012) 21-52.

[7] K. Y. Billah, S. R. H, Resonance, Tacoma Narrows bridge failure, and undergraduate physics textbooks, American Journal of Physics 59 (1991) 118-124. 
[8] J. G. S. da Silva, P. da S Vellasco, S. A. L. de Andrade, L. R. O. de Lima, F. P. Figueiredo, Vibration analysis of footbridges due to vertical human loads, Computers and Structures 85 (2007) 1693-1703.

[9] P. Dallard, T. Fitzpatrick, A. Flint, A. Low, R. R. Smith, M. Willford, M. Roche, London millennium bridge: Pedestrian-induced lateral vibration, Journal of Bridge Engineering, Trans. ASCE 6 (2001) 412-417.

[10] A. Kolozsváry, Roof arches without bending moments, patent, WO/2006/136867 (2006).

[11] K. Hincz, Arch-supported tensile structures with very long clear spans, International Association of Shell and Spatial Structures 48 (2007) 89-98.

[12] K. Hincz, Nonlinear analysis of cable net structures suspended from arches with block and tackle suspension system, taking into account the friction of the pulleys, International Journal of Space Structures 24 (2009) 143-152.

[13] K. Hincz, O. Baverel, Investigation of an arched footbridge funicular against any loads, in: IABSE-IASS 2011 Symposium, IABSE-IASS, London, UK, 2011, pp. 1-7.

[14] S. Timoshenko, D. H. Young, W. Weaver, Vibration Problems in Engineering, John Wiley \& Sons, New York, 1974.

[15] J. M. T. Thompson, A. R. Bokaian, R. Ghaffari, Subharmonic resonances and chaotic motions of a bilinear oscillator, IMA Journal of Applied Mathematics 31 (3) (1983) 207-234.

[16] F. C. Moon, S. W. Shaw, Chaotic vibrations of a beam with non-linear boundary conditions, International Journal of Non-linear Mechanics 18 (6) (1983) 465-477.

[17] S. Timoshenko, D. H. Young, Theory of structures, McGraw-Hill, New York, 1945.

[18] Maplesoft, Maple 9.03 Computer Algebra System, Waterloo, ON, Canada.

[19] R. E. D. Bishop, A. Y. Hassan, The lift and drag forces on a circular cylinder in a flowing fluid, Proceedings of the Royal Society A 277 (1964) 32-50.

[20] O. M. Griffin, G. H. Koopmann, The vortex-excited lift and reaction forces on resonantly vibrating cylinders, Journal of Sound and Vibration 54 (3) (1977) 435-448.

[21] T. Sarpkaya, Vortex-induced oscillations, Journal of Applied Mechanics 46 (1979) 241-258.

[22] O. M. Griffin, Vortex-excited cross-flow vibrations of a single cylindrical tube, Journal of Pressure Vessel Technology 102 (2) (1980) 158-166. 
[23] R. D. Gabbai, H. Benaroya, An overview of modeling and experiments of vortex-induced vibration of circular cylinders, Journal of Sound and Vibration 282 (2005) 575-616.

[24] C. H. K. Williamson, R. Govardhan, Vortex-induced vibrations, Annual Review of Fluid Mechanics 36 (2004) 413-455.

[25] G. Diana, F. Resta, M. Belloli, D. Rocchi, On the vortex shedding forcing on suspension bridge deck, Journal of Wind Engineering and Industrial Aerodynamics 94 (2006) 341-363.

[26] C. M. Harris, A. G. Piersol, Harris' Shock and Vibration Handbook, McGraw-Hill, New York, 2002.

[27] F. Beer, E. Russell Johnston Jr., J. T. DeWolf, D. F. Mazurek, Mechanics of Materials, McGraw-Hill, New York, 2009.

[28] FIB, Guidelines for the design of footbridges (November 2005).

[29] Sétra, Assessment of vibrational behaviour of footbridges under pedestrian loading, technical guide (October 2006).

[30] H. Bachmann, Case studies of structures with man-induced vibrations, Journal of Structural Engineering, Trans. ASCE 118 (1992) 631-647. 